\title{
NUMERICAL AND THEORETICAL STUDY OF PHOTONIC CRYSTAL FIBERS
}

\author{
S. Guenneau, A. Nicolet, F. Zolla, and S. Lasquellec
}

Institut Fresnel, UMR 6133, Faculté de Saint Jérôme case 162, 13397 Marseille Cedex 20, France

Abstract - In this work, we study a novel type of optical waveguide, whose properties derive from a periodic arrangement of fibers (not necessarily circular), and from a central structural defect along which the light is guided. We first look for propagating modes in photonic crystal fibers of high index core region which can be single mode at any wavelength [1-4]. Unlike the first type of photonic crystal fibers, whose properties derive from a high effective index, there exists some fundamentally different type of novel optical waveguides which consist in localizing the guided modes in air regions [4-5]. These propagating modes are localized in a low-index structural defect thanks to a photonic bandgap guidance for the resonant frequencies (coming from the photonic crystal cladding). We achieve numerical computations with the help of a new finite element formulation for spectral problems arising in the determination of propagating modes in dielectric waveguides and particularly in optical fibers [7]. The originality of the paper lies in the fact that we take into account both the boundness of the crystal (no Bloch wave expansion or periodicity boundary conditions) and the unboundness of the problem (no artificial boundary conditions at finite distance). We are thus led to an unbounded operator (open guide operator) and we must pay a special attention to its theoretical study before its numerical treatment. For this, we choose the magnetic field as the variable. It involves both a transverse field in the section of the guide and a longitudinal field along its axis. The section of the guide is meshed with triangles and Whitney finite elements are used, i.e., edge elements for the transverse field and node elements for the longitudinal field. To deal with the open problem, a judicious choice of coordinate transformation allows the finite element modeling of the infinite exterior domain. It is to be noticed that the discretization of the open guide operator leads to a generalized eigenvalue problem, solved thanks to the Lanczos algorithm. The code is validated by a numerical study of the classical 
cylindrical fiber for which the eigenmodes are known in closed form. We then apply the code to Low Index Photonic Crystal Fibers (LPCF) and to High Index Photonic Crystal Fibers (HPCF).

\section{Introduction}

\section{Setup of the Problem}

\section{Variational Methods and Finite Elements}

3.1 Notions of Differential Geometry

3.2 A Brief Survey on Edge Elements and Whitney Forms

3.3 A Discrete Analogue to the Variational Problem

3.4 Transformation Method

\section{Numerical Implementation}

4.1 The Lanczos Algorithm

4.2 Validation of the Code

4.3 Numerical Results for the PCF

\section{Conclusion}

\section{Acknowledgment}

\section{References}

\section{INTRODUCTION}

Optical fibers and integrated optical waveguides are today finding wide use in areas covering telecommunications, sensor technology, spectroscopy, and medicine. As was experimentally shown by Russell and co-workers [1-5], a special class of components incorporating photonic crystals can propagate light in a low index medium or be monomode at all wavelengths in a high index defect. More precisely, they fashioned a dielectric waveguide with a two-dimensional periodic variation in the plane perpendicular to the fiber axis, and an invariant structure along it. One application of such materials is a type of optical waveguide where light is confined by surrounding it with a band gap material. The photonic band gap effect (PBG) may be achieved in periodically structured materials having a periodicity on the scale of the optical wavelength: this is the well-known ability of photonic crystals to inhibit the propagation of photons with welldefined frequencies which has a close analogy with the electronic properties of semiconductors. Therefore, two-dimensional periodic 
structures in the form of long, fine silica fibers that have a regular array of tiny air holes running down their length [1-5] constitute twodimensional photonic crystals with lattice constants of the order of micrometers. In the sequel, we will refer to such structures as photonic crystal fibers (PCF). These microstructure fibers have recently been used to form both endlessly single-mode crystal fibers based on total internal reflection thanks to a high defect index core and the crystal cladding, lossless crystal fibers based on light confinement in a low defect index core by PBG effect. The first type of PCF is a glass fiber with a regular array of holes running down its length: we will call it in the sequel High Photonic Crystal Fiber (HPCF). A single missing hole in the array forms a region which effectively has a higher refractive index than the surrounding photonic crystal. This acts as a waveguide core in which light can become trapped, forming a guided mode. Russell et al [1-5] have experimentally demonstrated a singlemode operation in such waveguides in a wavelength range from 337 $\mathrm{nm}$ to $1550 \mathrm{~nm}$. Furthermore, it can be theoretically predicted that this type of PCF remains monomode even for very short wavelengths using the effective index model of Russell et al [1]. The first step is to note that in a standard step-index fiber with core radius $r$ and core and cladding indices $n_{c o}$ and $n_{c l}$, the number of guided modes is determined by the $V$ value [16]:

$$
V=\frac{2 \pi r}{\lambda} \sqrt{n_{c o}^{2}-n_{c l}^{2}}
$$

which must be less than 2.405 for the fiber to be single mode at optical wavelengths. Thus, single-mode fibers are in fact multimode for light of sufficiently short wavelength (with respect to the radius $r$ of the cavities). Russell et al [1] then propose an explanation for the monomode property of PCF based on the effective refractive index of the cladding. They find an empirical rule which determines whether the fiber is single mode or not:

$$
V_{\mathrm{eff}}=\frac{2 \pi d}{\lambda} \sqrt{n_{0}^{2}-n_{\mathrm{eff}}^{2}}
$$

where $d$ denotes the center-center spacing between holes, $n_{0}$ is the index of silica and

$$
n_{\mathrm{eff}}=\frac{\gamma_{\mathrm{eff}} \lambda}{2 \pi}
$$

$\gamma_{\text {eff }}$ being the maximum propagation constant $\gamma$ allowed in the cladding. $V_{\text {eff }}$ is then calculated by solving the scalar wave equation within a unit cell centered on one of the holes. Furthermore, they noted that for small $\lambda$, the scalar wave equation gives:

$$
\Lambda^{2} \Delta_{t} F+V_{\text {eff }}^{2} F=0,
$$


where $F$ stands for the electromagnetic field of the crystal in silica regions and $\Delta_{t}$ is the transverse part of the Laplacian. When $\lambda$ tends towards zero, the field $F$ is excluded from the low index air holes and is confined to the silica region bounded by the edges of the holes. For a given ratio of hole size to $\Lambda$, the field is therefore an invariant function of normalized transverse coordinates $\frac{x}{\Lambda}$ and $\frac{y}{\Lambda}$ in the short wavelength limit. The previous equation then implies that $V_{\text {eff }}$ is finite and independent of $\lambda$ and $\Lambda$ under these conditions. This situation contrasts with that for the step index fiber, for which $V$ tends to infinity as $\lambda$ tends to zero. Russell and co-workers then conclude that the limiting value of $V_{\text {eff }}$ depends on the relative size of the holes, but a sufficiently small value of $\lambda$ compared to the radius $r$ of the holes guarantees single-mode operation for all wavelengths $\lambda$ and scales $\Lambda$.

The second type of $\mathrm{PCF}$ is certainly the more surprising one, since it enables light propagation in a low index structural defect thanks to the PBG effect: we will call it in the sequel Low Photonic Crystal Fiber (LPCF). Its properties have been studied both theoretically and experimentally in honeycomb photonic crystal fibers made of around one hundred rods. For the numerical determination of the defect modes, Broeng et al [4] employed a super-cell enhancement of the plane-wave method (the basic cell contains the overall guide to take into account the defect). Let us recall that for a single mode, the fullvector wave equation of the magnetic field $H_{\mathbf{k}}$ may be obtained from Maxwell's equations and expressed as follows:

$$
\operatorname{curl} \frac{1}{\varepsilon_{r}} \operatorname{curl} H_{\mathbf{k}}=-\frac{\omega^{2}}{c^{2}} H_{\mathbf{k}}
$$

where $\mathbf{k}$ represents the wave propagation vector of the mode and $\varepsilon_{r}$ is the relative permittivity of the periodic structure. Taking advantage of the periodic nature of thc problem, the magnetic field may be expanded into a sum of plane waves using the Floquet-Bloch's theorem as follows:

$$
H_{\mathbf{k}}=\sum_{\mathbf{G}} h_{\mathbf{k}-\mathbf{G}} e^{-i(\mathbf{k}-\mathbf{G}) \cdot \mathbf{r}},
$$

where $\mathbf{G}$ represents a lattice vector in reciprocal space, describing the periodic structure. The dielectric constant may be expressed as a Fourier series expansion to obtain:

$$
\frac{1}{\varepsilon_{r}}=\sum_{\mathbf{G}} V_{\mathbf{G}} e^{i \mathbf{G} \cdot \mathbf{r}},
$$


where $V_{\mathbf{G}}$ denotes the following Fourier coefficients of $\frac{1}{\varepsilon_{r}}$ :

$$
V_{\mathbf{G}}=\frac{1}{\operatorname{meas}(Y)} \int_{Y} \frac{1}{\varepsilon_{r}} e^{-i \mathbf{G} \cdot \mathbf{r}} d \mathbf{r} .
$$

In this equation, $Y$ denotes the smallest basic cell that may be used to represent the cross-section of the guide to be periodized (and meas $(Y)$ the measure of $Y$, i.e., the area of $Y$ in 2D and its volume in 3D). Therefore, a matrix generalized eigenvalue problem is obtained, where, for a fixed wave vector $\mathbf{k}$, the frequencies $\omega$ of the allowed modes in the periodic structures are found as complex eigenvalues.

The main drawback of this method is that it is necessary to suppose a priori that the finite size of the crystal cladding has no influence on the propagating modes, which is far from being obvious. We will see in the sequel that such an assumption could lead to unphysical solutions: the bounded cross-section of the PCF induces a discrete spectrum which corresponds to propagating modes and the exterior of the photonic guide induces a continuous spectrum (nonpropagating modes). Therefore, one question naturally arises: how can we make a distinction between physical modes which belong to the discrete spectrum and that of the continuous one if we consider the operator associated to the Bloch-wave decomposition?

In this study, we try to give both theoretical and numerical elements to answer this question. To be complete, we must add that our modelling allows us only to observe non-dissipative modes: we therefore have numerical access to the first type of PCF (HPCF). Concerning the second type (LPCF), we outline an alternative approach to that of Broeng et al: although this numerical study is not yet achieved, we explain our battle plan to find such leaky modes.

\section{SETUP OF THE PROBLEM}

We consider a dielectric waveguide of constant section $\Omega$, invariant along the $z$ axis and whose permittivity profile $\varepsilon$ is supposed to be a known function (e.g., a piecewise constant function to modelize a photonic crystal fiber). We are looking for electromagnetic fields $(\mathcal{E}, \mathcal{H})$ solutions of the following Maxwell equations:

$$
\left\{\begin{aligned}
\operatorname{curl} \mathcal{H} & =\varepsilon \frac{\partial \mathcal{E}}{\partial t} \\
\operatorname{curl} \mathcal{E} & =-\mu_{0} \frac{\partial \mathcal{H}}{\partial t}
\end{aligned}\right.
$$

$\mu_{0}$ being the permeability of vacuum. 
Furthermore, choosing a time dependence in $e^{-i \omega t}$, and taking into account the invariance of the guide along its $z$ axis, we define time-harmonic two-dimensional electric and magnetic fields $\mathbf{E}$ and $\mathbf{H}$ by:

$$
\left\{\begin{aligned}
\mathcal{E}(x, y, z, t) & =\Re\left(\mathbf{E}(x, y) e^{-i(\omega t-\gamma z)}\right) \\
\mathcal{H}(x, y, z, t) & =\Re\left(\mathbf{H}(x, y) e^{-i(\omega t-\gamma z)}\right)
\end{aligned}\right.
$$

If $\left[L^{2}\left(\mathbb{R}^{2}\right)\right]^{3}$ denotes the Hilbert space of square integrable functions on $\mathbb{R}^{2}$ with values in $\mathbb{C}^{3}$, we say that $(\mathbf{E}, \mathbf{H})$ is a guided mode when:

$$
\left\{\begin{aligned}
(\gamma, \omega) & \in \mathbb{R}_{+}^{2} \\
(\mathbf{E}, \mathbf{H}) & \neq(\mathbf{0}, \mathbf{0}) \\
\mathbf{E}, \mathbf{H} & \in\left[L^{2}\left(\mathbb{R}^{2}\right)\right]^{3}
\end{aligned}\right.
$$

where $\omega$ is the angular frequency in the vacuum and $\gamma$ denotes the propagating constant of the guided mode.

For $(\mathbf{E}, \mathbf{H})$ satisfying (1), (2) can be written as:

$$
\left\{\begin{aligned}
\operatorname{curl}_{\gamma} \mathbf{H} & =-i \omega \varepsilon_{0} \varepsilon_{r}(x, y) \mathbf{E} \\
\operatorname{curl}_{\gamma} \mathbf{E} & =i \omega \mu_{0} \mathbf{H}
\end{aligned}\right.
$$

where $\varepsilon_{r}$ denotes the relative permittivity such that $1 \leq \varepsilon_{r} \leq \varepsilon^{+}=$ $\sup _{(x, y) \in \mathbb{R}^{2}} \varepsilon_{r}(x, y)$ (bounded and coercive function) and where $\operatorname{curl}_{\gamma} \mathbf{H}$ and $\operatorname{curl}_{\gamma} \mathbf{E}$ are defined by:

$$
\left\{\begin{aligned}
\operatorname{curl}_{\gamma} \mathbf{H}(x, y) & =\operatorname{curl}\left(\mathbf{H}(x, y) e^{i \gamma z}\right) e^{-i \gamma z} \\
\operatorname{curl}_{\gamma} \mathbf{E}(x, y) & =\operatorname{curl}\left(\mathbf{E}(x, y) e^{i \gamma z}\right) e^{-i \gamma z}
\end{aligned}\right.
$$

Furthermore, $\operatorname{div}_{\gamma}$ being an operator analogously defined to $\operatorname{curl}_{\gamma}$ in (4), it is clear that $\operatorname{div}_{\gamma} \operatorname{curl}_{\gamma} \varphi=0, \forall \varphi \in\left[\mathcal{D}\left(\mathbb{R}^{2}\right)\right]^{3}$, that is for smooth vector valued functions $\varphi$. Thus, denoting by $k_{0}$ the wave number $\omega \sqrt{\mu_{0} \varepsilon_{0}}$, we are led to the two following systems of Maxwell's type:

$$
\begin{gathered}
\left\{\begin{array}{l}
\operatorname{curl}_{\gamma} \operatorname{curl}_{\gamma} \mathbf{E}=k_{0}^{2} \varepsilon_{r} \mathbf{E} \\
\operatorname{div}_{\gamma}\left(\varepsilon_{0} \varepsilon_{r} \mathbf{E}\right)=0
\end{array}\right. \\
\left\{\begin{array}{l}
\operatorname{curl}_{\gamma}\left(\varepsilon_{r}^{-1} \operatorname{curl}_{\gamma} \mathbf{H}\right)=k_{0}^{2} \mathbf{H} \\
\operatorname{div}_{\gamma}\left(\mu_{0} \mathbf{H}\right)=0
\end{array}\right.
\end{gathered}
$$


It must be noticed that $\mathbf{H}$ (resp. E) can be deduced from $\left(4_{E}^{\prime}\right)$ (resp. $\left(4_{H}^{\prime}\right)$ ) thanks to (3.2) (resp. (3.1)). Making the obvious remark that the divergence of $\mathbf{H}$ is null, contrary to that of $\mathbf{E}$, we choose a magnetic formulation. We thus have the following lemma [8]:

Lemma 1 Let $s$ be a positive real. Then, the two following systems are equivalent in $\left[H^{1}\left(\mathbb{R}^{2}\right)\right]^{3}$ (space of functions in $\left[L^{2}\left(\mathbb{R}^{2}\right)\right]^{3}$ whose gradients are in $\left.\left[L^{2}\left(\mathbb{R}^{2}\right)\right]^{9}\right)$ :

$$
\begin{gathered}
\begin{cases}\operatorname{curl}_{\gamma}\left(\varepsilon_{r}^{-1} \operatorname{curl}_{\gamma} \mathbf{H}\right)=k_{0}^{2} \mathbf{H} & (5.1) \\
\operatorname{div}_{\gamma} \mathbf{H}=0 & (5.2)\end{cases} \\
\operatorname{curl}_{\gamma}\left(\varepsilon_{r}^{-1} \operatorname{curl}_{\gamma} \mathbf{H}\right)-s \nabla_{\gamma}\left(\operatorname{div}_{\gamma} \mathbf{H}\right)=k_{0}^{2} \mathbf{H}
\end{gathered}
$$

Noting that in $\mathbb{R}^{2} \backslash \bar{\Omega}$ (exterior of $\Omega$ ), (6) becomes:

$$
-\Delta \mathbf{H}+\gamma^{2} \mathbf{H}+(1-s) \nabla_{\gamma}\left(\operatorname{div}_{\gamma} \mathbf{H}\right)=k_{0}^{2} \mathbf{H},
$$

we take $s=1$ to get the vector Helmholtz equation outside $\Omega$. Our problem reduces to find real $\left(\gamma, \varepsilon_{r}\right)$ such that there exists $\mathbf{H}$ solution of the problem $(\mathcal{P})$ :

$$
\begin{cases}\mathbf{H} \in\left[H^{1}\left(\mathbb{R}^{2}\right)\right]^{3}, & \mathbf{H} \neq 0 \\ c\left(\gamma ; \mathbf{H}, \mathbf{H}^{\prime}\right)=k_{0}^{2}\left(\mathbf{H}, \mathbf{H}^{\prime}\right), & \forall \mathbf{H}^{\prime} \in\left[H^{1}\left(\mathbb{R}^{2}\right)\right]^{3}\end{cases}
$$

where $c(\gamma ; \cdot, \cdot)$ is the sesquilinear form defined by:

$$
c\left(\gamma ; \mathbf{H}, \mathbf{H}^{\prime}\right)=\int_{\mathbb{R}^{2}}\left(\frac{1}{\varepsilon_{r}} \operatorname{curl}_{\gamma} \mathbf{H} \cdot \overline{\operatorname{curl}_{\gamma} \mathbf{H}^{\prime}}+\operatorname{div}_{\gamma} \mathbf{H} \overline{\operatorname{div}_{\gamma} \mathbf{H}^{\prime}}\right) d x d y .
$$

Note that for all $\mathbf{U}$ in $\left[H^{1}\left(\mathbb{R}^{2}\right)\right]^{3}$, we have:

$$
\int_{\mathbb{R}^{2}}\left(\left|\operatorname{curl}_{\gamma} \mathbf{U}\right|^{2}+\left|\operatorname{div}_{\gamma} \mathbf{U}\right|^{2}\right) d x d y=\int_{\mathbb{R}^{2}}\left(|\nabla \mathbf{U}|^{2}+\gamma^{2}|\mathbf{U}|^{2}\right) d x d y,
$$

where $|\nabla \mathbf{U}|^{2}=\operatorname{Tr}\left((\nabla \mathbf{U})^{t} \nabla \mathbf{U}\right)=\left(\partial^{i i} \mathbf{U}_{i i}\right)^{2}$. Thus, asuming that $\inf _{(x, y) \in \mathbb{R}^{2}} \varepsilon_{r}(x, y)=1$, we deduce that $c(\gamma ; \cdot, \cdot)$ satisfies, $\forall \gamma \in \mathbb{R}^{+}, \forall \mathbf{H} \in$ $\left[H^{1}\left(\mathbb{R}^{2}\right)\right]^{3}$

$$
\left\{\begin{array}{l}
c(\gamma ; \mathbf{H}, \mathbf{H}) \geq \frac{1}{\varepsilon^{+}} \int_{\mathbb{R}^{2}}\left(|\nabla \mathbf{H}|^{2}+\gamma^{2}|\mathbf{H}|^{2}\right) d x d y \geq \frac{\min \left(\gamma^{2}, 1\right)}{\varepsilon^{+}}\|\mathbf{H}\|_{H^{1}\left(\mathbb{R}^{2}\right)}^{2} \\
c(\gamma ; \mathbf{H}, \mathbf{H}) \leq \int_{\mathbb{R}^{2}}\left(|\nabla \mathbf{H}|^{2}+\gamma^{2}|\mathbf{H}|^{2}\right) d x d y \leq \max \left(\gamma^{2}, 1\right)\|\mathbf{H}\|_{H^{1}\left(\mathbb{R}^{2}\right)}^{2}
\end{array}\right.
$$


where $\varepsilon^{+}$is given by $\varepsilon^{+}=\sup _{(x, y) \in \mathbb{R}^{2}} \varepsilon_{r}(x, y)$ and $\|\mathbf{H}\|_{H^{1}\left(\mathbb{R}^{2}\right)}^{2}=\int_{\mathbb{R}^{2}}\left(|\nabla \mathbf{H}|^{2}+\right.$ $\left.|\mathbf{H}|^{2}\right) d x d y$.

The system (7) ensures us that $c(\gamma)$ is thus continuous and coercive in $\left[H^{1}\left(\mathbb{R}^{2}\right)\right]^{3}$ if $\gamma \neq 0$. From Lax-Milgram lemma, we then deduce that (5) admits a unique solution in $\left[H^{1}\left(\mathbb{R}^{2}\right)\right]^{3}$ given by the minimum of the following functional in the Hilbert space $\left[H^{1}\left(\mathbb{R}^{2}\right)\right]^{3}$ :

$$
\begin{aligned}
\mathcal{R}\left(\gamma ; \mathbf{H}, \mathbf{H}^{\prime}\right)= & \int_{\mathbb{R}^{2}} \frac{1}{\varepsilon_{r}} \operatorname{curl}_{\gamma} \mathbf{H} \cdot \overline{\operatorname{curl}_{\gamma} \mathbf{H}^{\prime}} d x d y \\
& +s \int_{\mathbb{R}^{2}} \operatorname{div}_{\gamma} \mathbf{H} \overline{\operatorname{div}_{\gamma} \mathbf{H}^{\prime}} d x d y-k_{0}^{2} \int_{\mathbb{R}^{2}} \mathbf{H} \cdot \overline{\mathbf{H}^{\prime}} d x d y .
\end{aligned}
$$

Besides, from Lax-Milgram lemma we know that for a given positive real $\gamma$, there is a unique operator $C(\gamma)$ defined for all $\mathbf{H}$ and $\mathbf{H}^{\prime}$ in $\left[L^{2}\left(\mathbb{R}^{2}\right)\right]^{3}$ by $\left(C(\gamma) \mathbf{H}, \mathbf{H}^{\prime}\right)=c\left(\gamma, \mathbf{H}, \mathbf{H}^{\prime}\right)$. Thanks to $(7)$, it can be established that [8]:

Lemma 2 Let $\gamma$ be in $\mathbb{R}^{+}$. Then the operator $C(\gamma)$ is a self adjoint operator and its spectrum $\sigma(C(\gamma))$ satisfies the following inclusion:

$$
\sigma(C(\gamma)) \subset\left[\frac{\gamma^{2}}{\varepsilon^{+}},+\infty[\right.
$$

Besides, if we denote by $\sigma_{p}(C(\gamma))$ the set of eigenvalues of $C(\gamma)$, then $\frac{\gamma^{2}}{\varepsilon^{+}} \notin \sigma_{p}(C(\gamma))$.

We now develop $\mathbf{H}(x, y)$ in its transverse and longitudinal components $\mathbf{H}_{t}(x, y)$ and $H_{l}(x, y)$ :

$$
\begin{aligned}
\mathbf{H}(x, y) & =H_{t, 1}(x, y) \mathbf{e}_{x}+H_{t, 2}(x, y) \mathbf{e}_{y}+H_{l}(x, y) \mathbf{e}_{z} \\
& =\mathbf{H}_{t}(x, y)+H_{l}(x, y) \mathbf{e}_{z} .
\end{aligned}
$$

We define the transverse gradient, divergence and curl as

$$
\begin{aligned}
\nabla_{t} H_{l}(x, y) & =\frac{\partial H_{l}}{\partial x} \mathbf{e}_{x}+\frac{\partial H_{l}}{\partial y} \mathbf{e}_{y} \\
\operatorname{div}_{t}\left(H_{t, 1} \mathbf{e}_{x}+H_{t, 2} \mathbf{e}_{y}\right) & =\frac{\partial H_{t, 1}}{\partial x}+\frac{\partial H_{t, 2}}{\partial y} \\
\operatorname{curl}_{t}\left(H_{t, 1} \mathbf{e}_{x}+H_{t, 2} \mathbf{e}_{y}\right) & =\frac{\partial H_{t, 2}}{\partial x}-\frac{\partial H_{t, 1}}{\partial y}
\end{aligned}
$$


and we develop $\operatorname{curl}_{\gamma}$ and $\operatorname{div}_{\gamma}$ in their transverse and longitudinal components:

$$
\begin{aligned}
\operatorname{div}_{\gamma} \mathbf{H}= & \frac{\partial H_{t, 1}}{\partial x}+\frac{\partial H_{t, 2}}{\partial y}+i \gamma H_{l}=\operatorname{div}_{t} H_{t}+i \gamma H_{l} \\
\operatorname{curl}_{\gamma} \mathbf{H}= & \left(\frac{\partial H_{l}}{\partial y}-i \gamma H_{t, 2}\right) \mathbf{e}_{x}+\left(i \gamma H_{t, 1}-\frac{\partial H_{l}}{\partial x}\right) \mathbf{e}_{y} \\
& +\left(\frac{\partial H_{t, 2}}{\partial x}-\frac{\partial H_{t, 1}}{\partial y}\right) \mathbf{e}_{z} \\
= & \operatorname{curl}_{t} H_{t} \mathbf{e}_{z}+\left(\nabla_{t} H_{l}-i \gamma H_{t}\right) \times \mathbf{e}_{z}
\end{aligned}
$$

Making use of the Green formula:

$$
\int_{\mathbb{R}^{2}}\left(\nabla H_{l} \cdot \overline{\mathbf{H}}_{t}^{\prime}-\mathbf{H}_{t} \cdot \nabla \bar{H}_{l}^{\prime}\right) d x d y=\int_{\mathbb{R}^{2}}\left(\operatorname{div}_{t} \mathbf{H}_{t} \bar{H}_{l}^{\prime}-H_{l} \operatorname{div} \overline{\mathbf{H}}_{t}^{\prime}\right) d x d y,
$$

we derive the following lemma [8]:

Lemma 3 For all positive real $\gamma$, and for $\mathbf{H}$ and $\mathbf{H}^{\prime}$ in $\left[H^{1}\left(\mathbb{R}^{2}\right)\right]^{3}$, we have the following equality:

$$
c\left(\gamma, \mathbf{H}, \mathbf{H}^{\prime}\right)=d\left(\gamma, \mathbf{H}, \mathbf{H}^{\prime}\right)+\gamma^{2}\left(\mathbf{H}, \mathbf{H}^{\prime}\right),
$$

with $d(\gamma, \cdot, \cdot)$ defined by:

$$
d\left(\gamma, \mathbf{H}, \mathbf{H}^{\prime}\right)=d^{0}\left(\mathbf{H}, \mathbf{H}^{\prime}\right)+\gamma d^{1}\left(\mathbf{H}, \mathbf{H}^{\prime}\right)+\gamma^{2} d^{2}\left(\mathbf{H}, \mathbf{H}^{\prime}\right),
$$

where $d^{0}, d^{1}$ and $d^{2}$ are given by:

$$
\begin{aligned}
d^{0}\left(\mathbf{H}, \mathbf{H}^{\prime}\right)= & \int_{\mathbb{R}^{2}}\left(\frac{1}{\varepsilon_{r}} \operatorname{curl}_{t} \mathbf{H}_{t} \operatorname{curl}_{t} \overline{\mathbf{H}}_{t}^{\prime}+\operatorname{div}_{t} \mathbf{H}_{t} \operatorname{div}_{t} \overline{\mathbf{H}}_{t}^{\prime}\right. \\
& \left.+\frac{1}{\varepsilon_{r}} \nabla_{t} H_{l} \cdot \nabla_{t} \bar{H}_{l}^{\prime}\right) d x d y, \\
d^{1}\left(\mathbf{H}, \mathbf{H}^{\prime}\right)= & i \int_{\mathbb{R}^{2}}\left(\left(\frac{1}{\varepsilon_{r}}-1\right)\left(\mathbf{H}_{t} \cdot \nabla_{t} \bar{H}_{l}^{\prime}-\nabla_{t} H_{l} \cdot \overline{\mathbf{H}}_{t}^{\prime}\right)\right) d x d y, \\
d^{2}\left(\mathbf{H}, \mathbf{H}^{\prime}\right)= & \int_{\mathbb{R}^{2}}\left(\frac{1}{\varepsilon_{r}}-1\right) \mathbf{H}_{t} \cdot \overline{\mathbf{H}}_{t}^{\prime} d x d y .
\end{aligned}
$$

Remarks: From (9), we see that $c(\gamma)$ depends on both $\gamma$ and $\gamma^{2}$, i.e., $\varepsilon_{r}$ being fixed, the computation of $\gamma$ such that $\left(\gamma, \varepsilon_{r}\right)$ is a solution of $\mathcal{P}$, is a nonlinear problem. We thus choose to look for $\omega$ as a function of $\gamma$ (such an approach would not hold for a dispersive media). 
Let us also note that the functions $\gamma \in \mathbb{R}^{+} \longmapsto \Lambda_{m}(\gamma) \in \mathbb{R}^{+}$are monotonic, provided that

$$
\left(1-\frac{1}{\varepsilon^{+}}\right)\left(\sqrt{\varepsilon^{+}}+1\right)<1,
$$

which is a property shared by all the optical fibers [8]. The functions $\Lambda_{m}$ are therefore bijective on $\mathbb{R}^{+}$and the usual dispersion curve is straightforward from $(D R)$. To be more precise, the $\Lambda_{m}$ are even almost everywhere differentiable since they satisfy [8]:

$$
\frac{\left|\Lambda_{m}(\gamma)-\Lambda_{m}\left(\gamma^{\prime}\right)\right|}{\left|\gamma-\gamma^{\prime}\right|} \leq 2\left(1-\frac{1}{\varepsilon^{+}}\right)\left(\sqrt{\varepsilon^{+}}+1\right) \max \left(\gamma, \gamma^{\prime}\right) .
$$

We thus have an upper estimate for the slope of the curves: the lowest $\varepsilon^{+}$, the lowest the estimate (therefore the slope) and this estimate is asymptotically equal to $2\left(\sqrt{\varepsilon^{+}}+1\right) \max \left(\gamma, \gamma^{\prime}\right)$ for large $\varepsilon^{+}$. One cannot hope to show stronger properties on the derivatives of the curves, since there are some branches of eigenvalues crossing each other even in the case of a step profile optical fiber of circular cross section.

It is worth noting that we are dealing with an unbounded operator, since $d$ has a support in the overall space $\mathbb{R}^{2}$ (this would not be the case for a metallic guide of dielectric rods). We therefore have to deal with the essential part of the spectrum, that is the set of eigenvalues of infinite multiplicity, accumulation points of eigenvalues and the continuous spectrum (bands). It can be shown that for all $\gamma \in \mathbb{R}^{+}$, the essential spectrum $\sigma_{\mathrm{ess}}(C(\gamma))$ of $C(\gamma)$ satisfies [8]:

$$
\sigma_{\text {ess }}(C(\gamma))=\left[\gamma^{2} ;+\infty[.\right.
$$

We see that $d^{1}$ and $d^{2}$ are compact perturbations of $d^{0}$ (note that $\frac{1}{\varepsilon_{r}}-1$ is null outside $\Omega$ and conclude by the compact embedding of $\left[H^{1}(\Omega)\right]^{3}$ in $\left[L^{2}(\Omega)\right]^{3}$ ), thus they do not change its continuous spectrum $[9]$. We then have that:

$$
\sigma_{\mathrm{ess}}(C(\gamma))=\left\{\Lambda+\gamma^{2} ; \Lambda \in \sigma_{\mathrm{ess}}(C(0))\right\} .
$$

Furthermore, the Lemma 3 ensures us that $d^{0}(\mathbf{H}, \mathbf{H})$ satisfies for all $\mathbf{H} \in\left[H^{1}\left(\mathbb{R}^{2}\right)\right]^{3}$ :

$$
d^{0}(\mathbf{H}, \mathbf{H}) \geq \frac{1}{\varepsilon^{+}} \int_{\mathbb{R}^{2}}|\nabla \mathbf{H}|^{2} d x d y .
$$

Hence, we deduce that:

$$
\sigma_{\text {ess }}(C(0))=\mathbb{R}^{+} .
$$




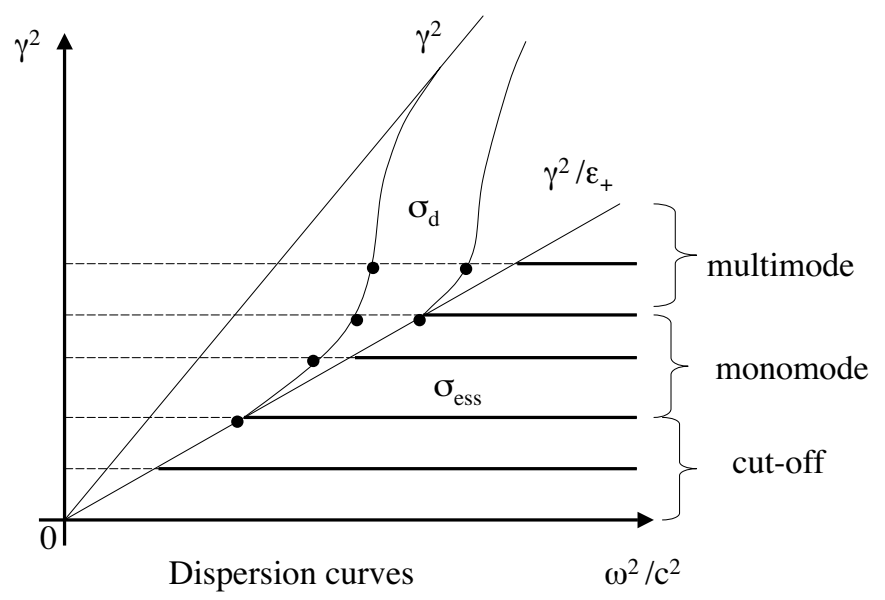

Figure 1. Decomposition of the spectrum $\sigma(C(\gamma))$ of the open guide operator $C(\gamma)$ in its discrete part $\sigma_{d}$ (which gives the dispersion curves when $\gamma$ varies in $\mathbb{R}_{\star}^{+}$) and its essential part $\sigma_{\text {ess }}$ (eigenvalues of infinite multiplicity, accumulation points of eigenvalues and continuous spectrum which are probably associated to the diffracting problem).

Furthermore, it can be proven that there are no eigenvalues in the essential spectrum exccpt for $\gamma^{2}[8]$ :

$$
\left.\left.\sigma_{d}(C(\gamma)) \subset\right]-\infty ; \gamma^{2}\right] .
$$

The operator $C(\gamma)$ is self adjoint, hence every point which is not in its essential spectrum is an isolated eigenvalue of finite multiplicity [9]. From Lemma 2, we thus derive that [8]:

Lemma 4 Every eigenvalue $\Lambda^{j}(\gamma), j \in\{1, \ldots, k\}$, of the operator $C(\gamma)$ satisfies the following dispersion relations $(D R)$ :

$$
\frac{\gamma^{2}}{\sup _{x \in \mathbb{R}^{2}} \varepsilon_{r}(x)}<\Lambda^{1}(\gamma) \leq \Lambda^{2}(\gamma) \leq \cdots \leq \Lambda^{k}(\gamma) \leq \gamma^{2} .
$$

Remarks: An empty discrete spectrum $\sigma_{d}\left(C_{\gamma}\right)$ corresponds to frequencies under the cut-off, and an integer $k=1$ to a monomode fiber (Fig. 1).

Thanks to the dispersion relations $(D R)$, we know that every eigenvalue greater than $\gamma^{2}$ belongs to the essential spectrum, which gives us a numerical criterion to eliminate the modes without any physical meaning. Nevertheless, we numerically detect some frequencies in the essential spectrum whose associated eigenvectors 
seem to satisfy all the properties of propagating modes (notably null divergence). We believe that those eigenvectors may give us information on the diffracting problem in conical incidence.

\section{VARIATIONAL METHODS AND FINITE ELEMENTS}

\subsection{Notions of Differential Geometry}

From a differential geometry point of view, the vector fields are the first order linear differential operators on functions. They have a vector space structure, one basis of which is the set $\left\{\frac{\partial \cdot}{\partial x^{i}}\right\}$ of partial derivatives with respect to coordinates. The action of a vector field $v$ on a function $f$ is noted $v(f)$. A 1 -form $\alpha$ is a linear map from vector fields $v$ to scalar functions $\alpha(v)$, also noted $\langle\alpha, v\rangle$ to emphasize duality (at one point of space, a vector field is represented by a vector and a 1-form by a covector, i.e., a linear map from vectors to real numbers). A special 1-form associated to a function $f$ is its differential $d f$ defined such that $d f(v)=v(f)$. One basis for the vector space of 1-forms is the set $\left\{d x^{i}\right\}$ of the differentials of the coordinates. A $p$-form $\omega$ is a multilinear totally skew symmetric map from $p$ vectors $v_{1}, \ldots, v_{p}$ to scalar functions $\omega\left(v_{1}, \ldots, v_{p}\right)$. Functions are identified with 0 -forms. In three-space only 0-, 1-, 2- and 3-forms are not identically equal to zero (because of the skew-symmetry). 0- and 3-form spaces are onedimensional vector spaces while 1 - and 2 -forms are three-dimensional vector spaces (neglecting of course the functional aspect where they are all infinite dimensional). From this point of view, scalar fields from vector analysis are 0 - or 3 -forms depending on their physical meaning: 0 -forms are pointwise relevant functions while 3 -forms are densities to be integrated on volumes. The vector fields from the vector analysis are 1-forms and 2-forms: 1-forms are integrands of line integrals while 2 -forms are flux densities. Operations on forms include the exterior or wedge product $\wedge$ that maps pairs of a $p$-form $\omega_{1}$ and a $q$-form $\omega_{2}$ on the $(p+q)$-form $\omega_{1} \wedge \omega_{2}$ defined by:

$$
\begin{aligned}
\left(\omega_{1} \wedge \omega_{2}\right)\left(v_{1}, \ldots, v_{p+q}\right)= & \frac{1}{p ! q !} \sum_{\pi \in S_{p+q}}\left[\operatorname{Sgn}(\pi) \omega_{1}\left(v_{\pi(1)}, \ldots, v_{\pi(p)}\right)\right. \\
& \left.\times \omega_{2}\left(v_{\pi(p+1)}, \ldots, v_{\pi(p+q)}\right)\right]
\end{aligned}
$$

where $\pi$ runs over the set of permutations of $p+q$ indices. The set $\left\{d x^{i_{1}} \wedge \cdots \wedge d x^{i_{p}}\right\}$ of the linearly independent exterior products of $p$ differentials of the coordinates is a basis for the $\frac{n !}{p !(n-p) !}$-dimensional vector space of $p$-forms. Any $p$-form can be expressed as a linear combination of such $p$-monomials. 
Another fundamental operation on forms is the exterior derivative $d$ that maps $p$-forms

$$
\omega=\frac{1}{p !} \sum_{i_{1}, \ldots, i_{p}=1}^{n} \omega_{i_{1}, \ldots, i_{p}} d x^{i_{1}} \wedge \cdots \wedge d x^{i_{p}},
$$

on $(p+1)$-forms

$$
d \omega=\frac{1}{p !} \sum_{i_{1}, \ldots, i_{p}=1}^{n}\left(d \omega_{i_{1}, \ldots, i_{p}}\right) \wedge d x^{i_{1}} \wedge \cdots \wedge d x^{i_{p}},
$$

where $d \omega_{i_{1}, \ldots, i_{p}}$ stands for the differential of $\omega_{i_{1}, \ldots, i_{p}}$ considered as a function.

From this definition it is obvious that the exterior derivative of a function $f$ is its differential $d f$. The opposite operation of the exterior derivative is the integration of a $n$-form $\omega=f\left(x^{1}, \ldots, x^{n}\right) d x^{1} \wedge \cdots \wedge d x^{n}$ on a $n$-dimensional domain $M$, which is defined by:

$$
\int_{M} \omega=\int_{\mathbb{R}^{n}} f\left(x^{1}, \ldots, x^{n}\right) d x^{1} \cdots d x^{n},
$$

where $f$ is supposed to be zero outside $M$.

These objects and operations only involve the topology and the differential structure of the ambient space, i.e., they are independent of any notion of angle and/or distance. Those notions are introduced by giving a metric $g$, i.e., a symmetric bilinear map from two vector fields $v, w$ to scalar functions numbers $g(v, w)$. The metric allows the definition of the Hodge star operator $\star$ which maps $p$-forms on $(n-p)$-forms where $n$ is the dimension of the ambient space. In local coordinates the star operator is defined for an exterior $p$-monomial by (using the Einstein summation convention on repeated indices):

$$
\star d x^{i_{1}} \wedge \cdots \wedge d x^{i_{p}}=g^{i_{1} j_{1}} \cdots g^{i_{p} j_{p}} d x^{j_{p+1}} \wedge \cdots \wedge d x^{j_{n}} \varepsilon_{j_{1}, \ldots, j_{n}} \frac{\sqrt{|g|}}{(n-p) !},
$$

where $\varepsilon_{j_{1}, \ldots, j_{n}}$ is the Levi-Civita symbol. If the matrix the elements of which are $g_{i j}=g\left(\partial \cdot / \partial x^{i}, \partial \cdot / \partial x^{j}\right)$ is considered, the $g^{i j}$ are the components of its inverse and $|g|=\operatorname{det}\left(g_{i j}\right)$ is its determinant. By linearity, the definition of the star operator may be extended to any form. In three-space, the Hodge star operator maps 0-forms on 3forms, 1-forms on 2-forms and conversely. This is why only functions and vector fields are used in the vector analysis of the three-space with the Euclidean metric. 


\subsection{A Brief Survey on Edge Elements and Whitney Forms}

Edge elements is a nickname for recently developed finite-element bases for vector fields, whose degrees of freedom are not to be interpreted as the components of some vector field at mesh nodes, but as circulations of the field along element edges. We will see in the sequel that they do not impose on the magnetic and electric fields $H$ and $E$ more continuity than physics requires: edge-element approximations of (let's say) $H$, will have tangential continuity across material interfaces, but its normal component will not be forced to (unwanted) continuity by the nature of finite-element interpolants, as it may happen with some node-based elements. Edge-elements are part of a discrete algebraic-geometric-differential structure of finiteelement shape functions invented by $\mathrm{H}$. Whitney [6] which assign degrees of freedom to simplices of a given mesh: nodes, edges, facets, tetrahedra. This structure, the so-called Whitney complex, closely matches a continuous structure made of four vector subspaces of $L^{2}$ and of three differential operators $\nabla$, curl, div, which is known as the de Rham complex. This complex is called an exact sequence if the image of each operator domain of this structure is exactly the kernel of the next operator. Clearly, this statement depends upon the topological properties of the domains such as connectivity assumptions. We thus choose the point of view of differential geometry in the sequel.

Consider a simplicial mesh on a three-dimensional manifold $D$, that is, a set of tetrahedra which 2 by 2 have in common either a full facet, or a full edge, or a node (vertex), or nothing, and whose set union is $D$. We also assume a numbering of the nodes $n$, so that edge $e$ or facet $f$ can alternatively be described by a list of node numbers. We call $\mathcal{T}, \mathcal{F}, \mathcal{E}, \mathcal{N}$ respectively, the sets of tetrahedra (or volumes), facets, edges and nodes which constitute the mesh. Hence an edge $e$ element of $\mathcal{E}$ will be denoted by the ordered set $\{i, j\}$ of its two extremities. A node $n$ element of $\mathcal{N}$ will be denoted by $\{i\}$ (i is the number of node $n$ ) and a facet $f$ element of $\mathcal{F}$ will be denoted by $\{i, j, k\}$. We shall adopt from now on the generic term of $p$-simplices to refer to nodes $(p=0)$, edges $(p=1)$, facets $(p=2)$ and volumes $(p=3)$ : more precisely, a $p$-simplex denotes the convex envelope of $p+1$ nodes, i.e., if $x_{i}$ denotes the location of the $i^{\text {th }}$ node, the $p$-simplex is the set $\left\{\sum_{i=1}^{p+1} \beta_{i} x_{i}, \beta_{i} \geq 0, \sum_{i=1}^{p+1} \beta_{i}=1\right\}$. Let us emphasize that if edge $\{i, j\}$ belongs to $\mathcal{E}$, or if facet $\{i, j, k\}$ belongs to $\mathcal{F}$, then $\{j, i\}$ does not belong to $\mathcal{E}$, and neither $\{k, i, j\},\{j, k, i\}$, etc., do belong to $\mathcal{F}$ : each $p$-simplex appears only once, with a definite orientation. We shall associate with this tesselation four finite-dimensional vector 


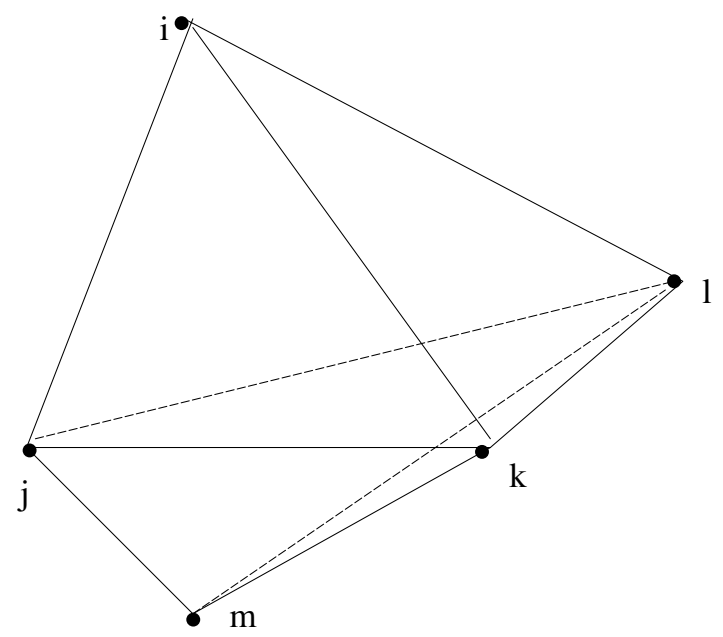

Figure 2. Two tetrahedra $\{i, j, k, l\}$ and $\{m, l, k, j\}$ with a common facet $\{j, k, l\}$.

spaces (i.e., discrete functional spaces), named $W^{p}, p=0$ to 3 , of $p$-forms (see Section 3.1 for a definition of $p$-forms). For each of these four vector spaces we may exhibit a basis (not an orthogonal one, but such that all base functions have a compact support). The elements of these bases are the node-, edge-, facet-, volume-elements alluded to.

Let us first define $\lambda_{i}^{j k l}(x)$ the barycentric coordinate of point $x$ with respect to node $\{\underline{i}\}$ located in $x_{\underline{i}}$ : this barycentric function is continuous over $D$, linear and null elsewhere. Consequently, this function is entirely defined by its values on the four vertices of the tetrahedra $\{\underline{i}, j, k, l\}$ :

$$
\left\{\begin{aligned}
\lambda_{i}^{j k l}\left(x_{i}\right) & =1 \\
\lambda_{i}^{j k l}\left(x_{m}\right) & =0, \quad \text { if } m \neq i .
\end{aligned}\right.
$$

Let us now denote by $K_{i}$ the set of integers $m$ such that $x_{m}$ and $x_{i}$ belong to the same edge. This way, we can define a function $\Lambda_{i}$ whose support is the set of tetrahedra which have the node $\left\{x_{i}\right\}$ in common (Fig. 2):

$$
\Lambda_{i}=\sum_{\{j, k, l\} \in K_{i}^{3}} \lambda_{i}^{j k l},
$$

(of course, in the case of curved tetrahedra, this should be understood in the reference space). We are now ready to define Whitney elements 
of order $p=0,1,2,3$. They are differential $p$-forms associated with $p$-simplices and generate the spaces $W^{p}$. For a node $n=\{i\}$, we set:

$$
w_{n}(x)=\Lambda_{i}(x) .
$$

This is a "Whitney element of order 0 ," based at node $n$. These elements are nothing else than the familiar piecewise linear, continuous $P^{1}$ elements: the "hat functions," or "Lagrange elements of polynomial degree 1," of finite element theory $\left(P^{k}\right.$ stands for the set of polynomial functions of degree $\leq k$ ). To edge $e=\{i, j\}$, we assign the "Whitney element of order 1," or "edge-element" based at edge $e$, defined by the 1 -form (vector field):

$$
w_{e}=\Lambda_{i} \nabla \Lambda_{j}-\Lambda_{j} \nabla \Lambda_{i} .
$$

To facet $f=\{i, j, k\}$, we assign the "facet-element" defined by the 2 -form (vector field):

$$
w_{f}=2\left(\Lambda_{i} \nabla \Lambda_{j} \times \nabla \Lambda_{k}+\Lambda_{k} \nabla \Lambda_{i} \times \nabla \Lambda_{j}+\Lambda_{j} \nabla \Lambda_{k} \times \nabla \Lambda_{i}\right) .
$$

Finally, we associate with volume $\{i, j, k, l\}$ a function whose analytical expression can be inferred from (12) and (13) (a factor 6 should be thrown in), but it is piecewise constant ( $P^{0}$ element), so writing it down would be pointless. The 1 -form in (12) is certainly not continuous over $D$, since the $\lambda_{i}$ s are not continuously differentiable. But it has some continuity nevertheless. To see it, consider the gradient (exterior derivative) of the 0 -form $\Lambda_{i}$ :

$$
\nabla \Lambda_{i}=\left\{\nabla \Lambda_{i}\right\}+n\left[\Lambda_{i}\right] \delta_{\Sigma},
$$

where $\delta_{\Sigma}$ denotes a Dirac mass with support on the tetrahedral facet opposite to the node $i,\left[\Lambda_{i}\right]$ denotes the jump of $\Lambda_{i}$ across this facet, $n$ denotes the outward normal to this facet and $\left\{\nabla \Lambda_{i}\right\}$ denotes the regular part of $\nabla \Lambda_{i}$. Thanks to the continuity of $\Lambda_{j}$, we both know that $\left[\Lambda_{j}\right]=0$ and $\left[\Lambda_{j} \nabla \Lambda_{i}\right]=\Lambda_{j}\left[\nabla \Lambda_{i}\right]$. If we then take the rotational of the 1 -form $\Lambda_{j} \nabla \Lambda_{i}$, we get that:

$$
\operatorname{curl}\left(\Lambda_{j} \nabla \Lambda_{i}\right)=\left\{\operatorname{curl}\left(\Lambda_{j} \nabla \Lambda_{i}\right)\right\}+n \Lambda_{j} \times\left[\nabla \Lambda_{i}\right] \delta_{\Sigma} .
$$

One cannot hope the continuity of $\nabla \Lambda_{i}$ on $\Sigma$ since it is not differentiable on $\Sigma$. Nevertheless, it is obvious from the definition of $\lambda_{i}^{j k l}$ :

$$
\lambda_{i}^{j k l}(x)=\sum_{p=1}^{3} \frac{n_{p}^{i j k l} x_{p}+a^{i j k l}}{n_{0}^{i j k l} x_{0}+a^{i j k l}},
$$


where $n_{p}^{i j k l}$ is the outward normal to the facet $\{j, k, l\}$ opposite to the node $\{i\}$ (tetrahedron $\{i, j, k, l\}$ ) and $a^{i j k l}$ is solely defined by (10). Besides $n_{p}^{i j k l}$ and $a^{i j k l}$ are the coefficients defining the plane (Fig. 2)

$$
\sum_{k=1}^{3} n_{p}^{i j k l} x_{k}+a^{i j k l}=0,
$$

such that $\nabla \Lambda_{i}$ is colinear to the outward normal $n$ of the previous plane (whose components are given by the $n_{p}^{i j k l}$ ). After tedious but easy computations, the reader can check that for each facet $n \times\left[\nabla \lambda_{i}\right]=0$.

We can thus conclude that the rotational (exterior derivative) of the 1 -form $w_{e}$ is given by:

$$
\begin{aligned}
\operatorname{curl} w_{e} & =\Lambda_{i} \operatorname{curl} \nabla \Lambda_{j}+\nabla \Lambda_{i} \times \nabla \Lambda_{j}-\Lambda_{j} \operatorname{curl} \nabla \Lambda_{i}-\nabla \Lambda_{j} \times \nabla \Lambda_{i} \\
& =2 \nabla \Lambda_{i} \times \nabla \Lambda_{j} .
\end{aligned}
$$

The very fact that $\operatorname{curl} w_{e}$ exists as 2-form, and not as a distribution proves that the tangential part of $w_{e}$ on facets of the mesh is continuous across these facets [10]. In other words, the tangential component of $w_{e}$ on a common facet $\{j, k, l\}$ of two adjacent tetrahedra $\{i, j, k, l\}$ and $\{m, l, k, j\}$ depends only on its values on the three edges $\{j, k\},\{k, l\},\{l, i\}$ (Fig. 2).

From this remark, we can already infer that Whitney vector fields of degree 1 (that is, those generated by edge-elements) are exactly what is needed in order to represent vector field like $\mathbf{E}$ (electric field) or $\mathbf{H}$ (magnetic field) in electromagnetic computations: for these fields (1-forms) have precisely this kind of continuity, i.e., continuity of the tangential part across material interfaces.

A similar remark holds about facet-elements $(p=2)$. For this, let us consider the divergence of (13). We first note that the Poynting identity ensures us that:

$$
\operatorname{div}\left(\Lambda_{i} \nabla \Lambda_{j} \times \nabla \Lambda_{k}\right)=\nabla \Lambda_{k} \cdot \operatorname{curl}\left(\Lambda_{i} \nabla \Lambda_{j}\right)-\Lambda_{i} \nabla \Lambda_{j} \cdot \operatorname{curl}\left(\nabla \Lambda_{k}\right) .
$$

If we develop the previous equation, we get that:

$$
\begin{aligned}
\operatorname{div}\left(\Lambda_{i} \nabla \Lambda_{j} \times \nabla \Lambda_{k}\right)= & \nabla \Lambda_{k} \cdot\left(\Lambda_{i} \operatorname{curl} \nabla \Lambda_{j}+\nabla \Lambda_{i} \times \nabla \Lambda_{j}\right) \\
& -\Lambda_{i} \nabla \Lambda_{j} \cdot \operatorname{curl}\left(\nabla \Lambda_{k}\right) .
\end{aligned}
$$

We have already seen that $\operatorname{curl} \nabla \Lambda_{j}=\left\{\operatorname{curl} \nabla \Lambda_{j}\right\}=0$. We therefore deduce that:

$$
\operatorname{div} w_{f}=6 \nabla \Lambda_{i} \cdot\left(\nabla \Lambda_{j} \times \nabla \Lambda_{k}\right),
$$


which is a 3 -form. Besides, we can write the exterior derivative of the 2 -form $w_{f}$ in terms of continuous form and de Rham current as follows:

$$
\operatorname{div} w_{f}=\left\{\operatorname{div} w_{f}\right\}+n \cdot\left[w_{f}\right] \delta_{\Sigma} .
$$

We hence conclude that the normal component $n \cdot w_{f}$ of $w_{f}$ is thus continuous across facets. The facet-elements are thus closely related to the magnetic and electric flux densities $B$ and $D$ (2-forms). It is worth noting that the tangential continuity of $w_{e}$ and the normal continuity of $w_{f}$ reveal the deep nature of Whitney elements which are built to ensure their continuity and that of their exterior derivative. Furthermore, $w_{e}$ satisfies

$$
\begin{aligned}
\operatorname{div}\left(\operatorname{curl} w_{e}\right) & =\operatorname{div}\left(\nabla \Lambda_{i} \times \nabla \Lambda_{j}\right) \\
& =\nabla \Lambda_{j} \cdot \operatorname{curl} \nabla \Lambda_{i}-\nabla \Lambda_{i} \cdot \operatorname{curl}\left(\nabla \Lambda_{j}\right)=0,
\end{aligned}
$$

which is nothing else than $d d w=0$. Moreover, using differential forms, we are able to exhibit a general expression for Whitney forms [11]:

$$
\omega_{s}=\sum_{i=0}^{p}(-1)^{i} \lambda_{i} d \lambda_{0} \wedge \cdots \wedge d \lambda_{i-1} \wedge d \lambda_{i+1} \wedge \cdots \wedge d \lambda_{p} .
$$

Now let us consider $p$-forms built from Whitney elements, i.e., following linear combinations:

$$
u=\sum_{s \in \mathbf{S}} \bar{u}_{s} w_{s}
$$

where the set $\mathbf{S}$ of $p$-simplices is $\mathcal{N}, \mathcal{E}, \mathcal{F}$ or $\mathcal{T}$, according to the value $p$. If $p=0$ (thus $\mathbf{S}=\mathcal{N}$, the set of nodes), the $\bar{u}_{s}$ are the nodal values of $u$, hence a clear interpretation of the degrees of freedom $\bar{u}_{s}$. If $p=1$ (thus $\mathbf{S}=\mathcal{E}$ ), it follows from the very definition of $w_{e}(12)$ that its circulation along edge $e$ (from node $\{i\}$ to node $\{j\}$ ) is equal to 1 , and to 0 along the other edges. Therefore, in the development of $u$, the degree of freedom $\bar{u}_{s}$ is the nodal value of $u$ if $s$ is a node, and the circulation of $u$ along the edge, if $s$ is an edge.

Furthermore, $\lambda_{i}(x)$ being the barycentric coordinate of point located in $x_{i}$, it must satisfy:

$$
\sum_{i} \lambda_{i}(x)=1, \quad \forall x \in D
$$

where $i$ denotes the nodes of $D$. This and (12) imply that:

$$
\sum_{j \neq i} w_{\{i, j\}}=\nabla \Lambda_{i}, \quad \text { for all nodes } i \text { and } j \text { of } D .
$$


Therefore, the gradient of a 0 -Whitney element is a linear combination of 1-Whitney elements, which we may express as follows:

$$
\nabla W^{0} \subset W^{1} .
$$

Similarly, one can prove that $\operatorname{curl} W^{1} \subset W^{2}$ and $\operatorname{div} W^{2} \subset W^{3}$. We can summarize these injectivity properties of the Whitney complex in the following diagram:

$$
W^{0} \stackrel{\nabla}{\longrightarrow} W^{1} \stackrel{\text { curl }}{\longrightarrow} W^{2} \stackrel{\text { div }}{\longrightarrow} W^{3} .
$$

We can express the same phenomena in terms of spaces $\bar{W}^{p}$ of degrees of freedom:

$$
\bar{W}^{0} \stackrel{G}{\longrightarrow} \bar{W}^{1} \stackrel{C}{\longrightarrow} \bar{W}^{2} \stackrel{D}{\longrightarrow} \bar{W}^{3},
$$

where $\bar{W}^{0}$ is the set of the node's values of the field, $\bar{W}^{1}$ is the set of the circulation along the edges, $\bar{W}^{2}$ is the set of the flow across the facets and $\bar{W}^{3}$ is the set of the volume integral on the elements. The symbols $G, C, D$ denote rectangular incidence matrices (whose entries are all 0,1 or -1 ) which represent $\nabla$, curl, div in the bases provided by Whitney elements. For instance, if $h=\sum_{e \in \mathcal{E}} \bar{h}_{e} w_{e}$, then $\operatorname{curl} h=\sum_{f \in \mathcal{F}} C_{f_{e}} \bar{h}_{e} w_{f}$ : the size of $C$ is the number of facets times the number of edges. If we denote by $N_{n}, N_{e}, N_{f}$ and $N_{v}$ respectively the number of nodes $\left(N_{n}=\right.$ Card $\left.\mathcal{N}\right)$, edges, facets and volumes, one can see that $G$ and $D$ are similarly matrices with $N_{e}$ lines and $N_{n}$ column, and $N_{v}$ lines and $N_{f}$ column. The fundamental properties of these matrices, as shown by the diagram are $C G=0$ and $D C=0$ : there is an isomorphism between the $W^{p}$ and the $\bar{W}^{p}$, which are spanned by the vectors of degrees of freedom. We will see in the sequel that the magnetic field $H$ actually "lives" in the space of 1-forms and is therefore well represented at the discrete level as an element of $W^{1}$ given by the set of coefficients $\bar{W}^{1}$ (to be determined by our numerical method).

\subsection{A Discrete Analogue to the Variational Problem}

The electromagnetic fields and sources may be represented by differential forms: the magnetic field $\mathbf{H}$ and the electric field $\mathbf{E}$ are 1-forms, the magnetic flux density $\mathbf{B}$, the electric flux density $\mathbf{D}$ and the current density $\mathbf{J}$ are 2 -forms and the charge density $\rho$ is a 3 -form. In this 
representation, the vector Maxwell system is given by:

$$
\left\{\begin{aligned}
d \mathbf{H} & =\mathbf{J}+\frac{\partial \mathbf{D}}{\partial t} \\
d \mathbf{E} & =-\frac{\partial \mathbf{B}}{\partial t} \\
d \mathbf{B} & =0 \\
d \mathbf{D} & =\rho
\end{aligned}\right.
$$

The 1-form $\mathbf{A}$ and the 0 -form $V$ may be introduced as potentials such that $\mathbf{B}=d A$ and $\mathbf{E}=-\frac{\partial \mathbf{A}}{\partial t}-d V$. All those equations are obviously independent of the metric. Nevertheless, this one is involved in the definition of the constitutive relations given by $\mathbf{D}=\varepsilon \star \mathbf{E}$ and $\mathbf{B}=\mu_{0} \star \mathbf{H}$.

In this representation, the time harmonic vector Maxwell system is given by:

$$
\left\{\begin{array}{l}
d \mathbf{H}=-i \omega \varepsilon \star \mathbf{E} \\
d \mathbf{E}=i \omega \mu \star \mathbf{H} \\
d\left(\mu_{0} \star \mathbf{H}\right)=0 \\
d(\varepsilon \star \mathbf{E})=0
\end{array}\right.
$$

in the absence of current density and volumic charges.

In the case of a two-dimensional electromagnetic problem, invariant by translation along the $z$-axis, the geometry is described by the trace of the electric and magnetic fields on the transverse plane. We choose the magnetic field as the variable and we develop $\mathbf{H}(x, y)$ in its transverse and longitudinal components $\mathbf{H}_{t}(x, y)$ and $H_{l}(x, y)$ :

$$
\begin{aligned}
\mathbf{H}(x, y) & =H_{t, 1}(x, y) d x+H_{t, 2}(x, y) d y+H_{l}(x, y) d z \\
& =\mathbf{H}_{t}(x, y)+H_{l}(x, y) d z .
\end{aligned}
$$

If we take the exterior derivative to the 1 -form $\mathbf{H}_{t}$, we get that:

$$
d \mathbf{H}_{t}=\frac{\partial H_{t, 1}}{\partial y} d y \wedge d x+\frac{\partial H_{t, 2}}{\partial x} d x \wedge d y=\left(-\frac{\partial H_{t, 1}}{\partial y}+\frac{\partial H_{t, 2}}{\partial x}\right) d x \wedge d y .
$$

Applying the star Hodge operator $\star$ to $\mathbf{H}_{t}$, we get that:

$$
\star \mathbf{H}_{t}=H_{t, 1} d y \wedge d z+H_{t, 2} d z \wedge d x .
$$

If we take the exterior derivative of the previous expression, we obtain:

$$
d\left(\star \mathbf{H}_{t}\right)=\frac{\partial H_{t, 1}}{\partial x} d x \wedge d y \wedge d z+\frac{\partial H_{t, 2}}{\partial y} d y \wedge d z \wedge d x
$$




$$
=\left(\frac{\partial H_{t, 1}}{\partial x}+\frac{\partial H_{t, 2}}{\partial y}\right) d x \wedge d y \wedge d z=\left(\frac{\partial H_{t, 1}}{\partial x}+\frac{\partial H_{t, 2}}{\partial y}\right) \star 1 .
$$

We define the transverse gradient, divergence and curl as follows:

$$
\begin{aligned}
\nabla_{t} H_{l}(x, y) & =d H_{l}=\frac{\partial H_{l}}{\partial x} d x+\frac{\partial H_{l}}{\partial y} d y \\
\operatorname{div}_{t}\left(\mathbf{H}_{t}\right) & =\star d \star \mathbf{H}_{t}=\frac{\partial H_{t, 1}}{\partial x}+\frac{\partial H_{t, 2}}{\partial y} \\
\operatorname{curl}_{t}\left(\mathbf{H}_{t}\right) d z & =\star d \mathbf{H}_{t}=\left(\frac{\partial H_{t, 2}}{\partial x}-\frac{\partial H_{t, 1}}{\partial y}\right) d z
\end{aligned}
$$

We can thus rewrite the problem $\left(4_{H}^{\prime}\right)$ with the formalism of differential forms as follows:

$$
\left\{\begin{array}{l}
\star \operatorname{curl}_{\gamma}\left(\varepsilon_{r}^{-1} \star \operatorname{curl}_{\gamma} \mathbf{H}\right)=k_{0}^{2} \mathbf{H} \\
\operatorname{div}_{\gamma}\left(\mu_{0} \star \mathbf{H}\right)=0
\end{array}\right.
$$

The discretization of the problem is based on Whitney forms. Assuming some Dirichlet boundary conditions on the boundary $\partial \theta$ of the three-dimensional manifold $\theta=\Omega \times \mathbb{R}$, we derive from $\left(4_{H}^{\prime}\right)$ that:

$$
\begin{aligned}
\int_{\theta} \star \star \varepsilon_{r}^{-1} \star \operatorname{curl}_{\gamma} \mathbf{H} \wedge \overline{\operatorname{curl}_{\gamma} \mathbf{H}^{\prime}} & =\int_{\theta} \varepsilon_{r}^{-1} \star \operatorname{curl}_{\gamma} \mathbf{H} \wedge \overline{\operatorname{curl}_{\gamma} \mathbf{H}^{\prime}} \\
& =k_{0}^{2} \int_{\theta} \star \mathbf{H} \wedge \overline{\mathbf{H}}^{\prime}, \quad \forall \mathbf{H} \in W_{0}^{1}
\end{aligned}
$$

where $W_{0}^{p}$ denotes the restriction of the space of $p$-forms on a finite element space $W^{p}$, with null value on $\partial \theta$.

Analogously to [10], we note that the sequence of $W^{p}$ is exact, i.e., that the image of $W^{p-1}$ by the operator $\nabla_{\gamma}\left(\operatorname{resp} . \operatorname{curl}_{\gamma}\right.$, $\left.\operatorname{div}_{\gamma}\right)$ is exactly the kernel of the next operator, thanks to the nullity of its trace (resp. the tangential and the normal trace). It is thus identified to the De Rham complex [10]. Let $\varphi^{\prime}$ be a test function in $W^{0}$. Noting that $\nabla_{\gamma} W^{0} \subset W^{1}$, we take $\mathbf{H}^{\prime}$ such that $\mathbf{H}^{\prime}=\nabla_{\gamma} \varphi^{\prime}$ and derive from (16) that (if $\omega \neq 0$ ):

$$
\int_{\theta} \mu_{0} \star \mathbf{H} \wedge \nabla_{\gamma} \overline{\varphi^{\prime}}=0, \quad \forall \varphi^{\prime} \in W^{0} .
$$

Bossavit has proved that this property solves the spurious mode problem for resonant cavities [10]. This weak formulation of null divergence $\operatorname{div}_{\gamma}\left(\mu_{0} \star \mathbf{H}\right)=0$ still works if $\mu_{0}$ is not constant. Therefore, 
replacing $\mathbf{H}$ by $\mathbf{E}$ and $\mu_{0}$ by $\varepsilon_{0} \varepsilon_{r}$, we see that Whitney forms clearly satisfy $\operatorname{div}_{\gamma}\left(\varepsilon_{0} \varepsilon_{r} \star \mathbf{E}\right)=0$. The electric formulation is thus straightforward from (16).

Concerning our formulation, we are dealing with $\mathbf{H} \in\left[H^{1}\left(\mathbb{R}^{2}\right)\right]^{3}$ which implies that $\mathbf{H}$ is null at infinity. The sequence of $W^{p}$ remaining exact (the trace conditions are obviously satisfied), the edge elements still fulfill (17).

We now develop $\operatorname{curl}_{\gamma}$ and $\operatorname{div}_{\gamma}$ in their transverse and longitudinal components:

$$
\begin{aligned}
\operatorname{div}_{\gamma} \mathbf{H}= & \frac{\partial H_{t, 1}}{\partial x}+\frac{\partial H_{t, 2}}{\partial y}+i \gamma H_{l}=\operatorname{div}_{t} H_{t}+i \gamma H_{l} \\
\operatorname{curl}_{\gamma} \mathbf{H}= & \left(\frac{\partial H_{l}}{\partial y}-i \gamma H_{t, 2}\right) d x+\left(i \gamma H_{t, 1}-\frac{\partial H_{l}}{\partial x}\right) d y \\
& +\left(\frac{\partial H_{t, 2}}{\partial x}-\frac{\partial H_{t, 1}}{\partial y}\right) d z \\
= & \operatorname{curl}_{t} H_{t} d z+\left(\nabla_{t} H_{l}-i \gamma H_{t}\right) \wedge d z
\end{aligned}
$$

If we use the star Hodge operator property $\star d z=d x \wedge d y$, we deduce that:

$$
\begin{aligned}
\star \operatorname{curl}_{\gamma} H \wedge \overline{\operatorname{curl}_{\gamma} H^{\prime}=} & \star\left(\operatorname{curl}_{t} H_{t} d z+\left(\nabla_{t} H_{l}-i \gamma H_{t}\right) \wedge d z\right) \\
& \wedge\left(\operatorname{curl}_{t} \overline{H_{t}^{\prime}} d z+\left(\nabla_{t} \overline{H_{l}^{\prime}}+i \gamma \overline{H_{t}^{\prime}}\right) \wedge d z\right) \\
= & \operatorname{curl}_{t} H_{t} \operatorname{curl}_{t} \overline{H_{t}^{\prime}} \star 1 \\
& +\star\left(\nabla_{t} H_{l}-i \gamma H_{t}\right) \wedge\left(\nabla_{t} \overline{H_{l}^{\prime}}+i \gamma \overline{H_{t}^{\prime}}\right) \\
= & \operatorname{curl}_{t} H_{t} \operatorname{curl}_{t} \overline{H_{t}^{\prime}} \star 1+\star \nabla_{t} H_{l} \wedge \nabla_{t} \overline{H_{l}^{\prime}} \\
& -i \gamma \star H_{t} \wedge \nabla_{t} \overline{H_{l}^{\prime}}+i \gamma \star \nabla_{t} H_{l} \wedge \overline{H_{t}^{\prime}} \\
& +\gamma^{2} \star H_{t} \wedge \overline{H_{t}^{\prime}} .
\end{aligned}
$$

From a numerical point of view, we minimize the following functional, which is a discrete analogue to (8):

$$
\begin{aligned}
\mathcal{R}\left(\gamma ; \mathbf{H}, \mathbf{H}^{\prime}\right)= & \int_{\theta} \varepsilon_{r}^{-1} \star\left(\operatorname{curl}_{t} \mathbf{H}_{t} \operatorname{curl}_{t} \overline{\mathbf{H}_{t}^{\prime}}+\nabla_{t} H_{l} \wedge \nabla_{t} \overline{H_{l}^{\prime}}\right. \\
& \left.-i \gamma \mathbf{H}_{t} \wedge \nabla_{t} \overline{H_{l}^{\prime}}+i \gamma \nabla_{t} H_{l} \wedge \overline{\mathbf{H}_{t}^{\prime}}+\gamma^{2} \mathbf{H}_{t} \wedge \overline{\mathbf{H}_{t}^{\prime}}\right) \\
& -k_{0}^{2} \int_{\theta} \star\left(\mathbf{H}_{t} \wedge \overline{\mathbf{H}_{t}^{\prime}}+H_{l} \overline{H_{l}^{\prime}}\right) .
\end{aligned}
$$

This formulation involves both a transverse field in the section of the guide and a longitudinal field along its axis. The section of the 
guide is meshed with triangles and Whitney finite elements are used, i.e., edge elements for the transverse field and node elements for the longitudinal field:

$$
\mathbf{H}= \begin{cases}\mathbf{H}_{t}=\sum_{j}^{n} \alpha_{j} \mathbf{w}_{\mathbf{j}}^{\mathbf{e}}(x, y) e^{i \gamma z}, & \text { in } \mathbb{R}^{2} \\ H_{z}=\sum_{j}^{n} \beta_{j} w_{j}^{n}(x, y) e^{i \gamma z}, & \text { in } \mathbb{R}\end{cases}
$$

where $\alpha_{j}$ denotes the line integral of the transverse component $\mathbf{H}_{t}$ on the edges, and $\beta_{j}$ denotes the line integral of the longitudinal component $H_{z}$ along one unit of length of the axis of the guide (which is equivalent to the nodal value). Besides, $\mathbf{w}_{\mathbf{j}}^{\mathbf{e}}$ and $w_{j}^{n}$ are respectively the basis functions of Whitney 1-forms and Whitney 0-forms.

\subsection{Transformation Method}

To deal with the open problem, a judicious choice of coordinate transformation allows the finite element modeling of the infinite exterior domain [12]. Considering two disks $D(O, A)$ and $D(O, B)$ of center $O=(0,0)$ and radii $A$ and $B>A$ strictly including $\Omega$, we define a corona $C=D(O, B) \backslash \overline{D(O, A)}$. Let $(x, y)$ be a point in $\mathbb{R}^{2} \backslash \overline{D(O, A)}$ (the infinite outer domain) and $(X, Y)$ be a point in $C$, the transformation is then given by:

$$
\begin{aligned}
& x=f_{1}(X, Y)=X[A(B-A)] /[R(B-R)], \\
& y=f_{2}(X, Y)=Y[A(B-A)] /[R(B-R)],
\end{aligned}
$$

where $R$ denotes the Euclidean norm $\sqrt{X^{2}+Y^{2}}$. This transformation may be viewed as a mapping of the finite corona $C$ with non orthogonal coordinate system $(X, Y)$ to the infinite domain with cartesian coordinate system $(x, y)$.

This way, the finite element discretization appears as a chained map from the reference space to the transformed space and from the transformed space to the physical space. Using discretizations entirely based on differential forms allows a straightforward formulation of transformation methods by pull-back of the corresponding weighted residuals. Following the remark of the preceding section, we fix $\gamma \in \mathbb{R}^{+}$and look for $(\omega, H)$ satisfying (8). We are thus led to a linear generalized eigenvalue system.

It is also to be noticed that taking Dirichlet boundary conditions at a finite distance (without geometric transformation) from the cross section of the guide allows to consider an operator with a compact resolvent (thus artificially eliminates the continuous spectrum) but it 


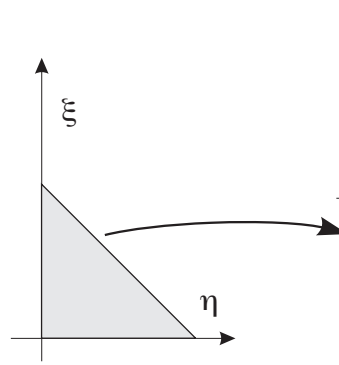

(a)

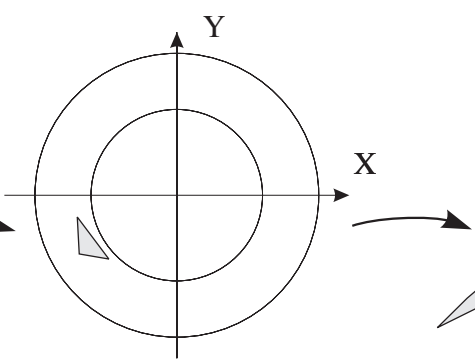

(b)

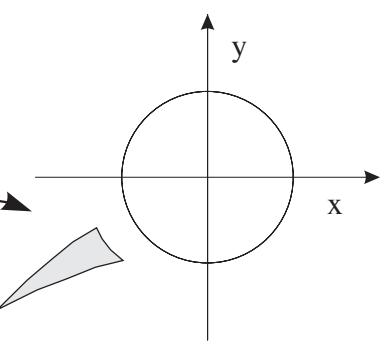

(c)

Figure 3. (a) Reference element. (b) Meshing of the corona equivalent to the unbounded domain with transformed coordinates. (c) Physical unbounded domain in cartesian coordinates.

would add the modes of an unphysical metallic guide. As for us, we pay a special attention to the unboundness of our resolvent operator (and hence the lack of compacity): the operator remains unbounded under the transformation method and we therefore use the relations $(D R)$ as a practical criterion to select the discrete spectrum corresponding to propagating modes and reject the numerical values corresponding to the continuous spectrum.

Remarks: Another way to discretize the problem would be to use vector nodal elements. The main drawback of this method is that vector nodal elements do not contain the gradients of the scalar nodal elements. One way of tackling this problem is thus to penalize the divergence. For this, we develop the divergence in its transverse and longitudinal components as follows:

$$
\begin{aligned}
\operatorname{div}_{\gamma} H \overline{\operatorname{div}_{\gamma} V}= & \operatorname{div}_{t} H_{t} \operatorname{div}_{t} \overline{H_{t}^{\prime}}+\gamma^{2} H_{l} \overline{H_{l}^{\prime}} \\
& +i \gamma\left(H_{l}\left(\operatorname{div}_{t} \overline{H_{t}^{\prime}}\right)-\left(\operatorname{div}_{t} H_{t}\right) \overline{H_{l}^{\prime}}\right) .
\end{aligned}
$$

The problem of minimization (8) therefore admits a unique solution thanks to the added term

$$
\begin{aligned}
s \int_{\theta} \operatorname{div}_{\gamma} \mathbf{H} \overline{\operatorname{div}_{\gamma} \mathbf{H}^{\prime}}= & s \int_{\theta}\left(\operatorname{div}_{t} H_{t} \operatorname{div}_{t} \overline{H_{t}^{\prime}}+\gamma^{2} H_{l} \overline{H_{l}^{\prime}}\right. \\
& \left.+i \gamma\left(H_{l}\left(\operatorname{div}_{t} \overline{H_{t}^{\prime}}\right)-\left(\operatorname{div}_{t} H_{t}\right) \overline{H_{l}^{\prime}}\right)\right),
\end{aligned}
$$

which acts in fact as a constraint which forces the nullity of the magnetic field divergence (the greater the positive real $s$, the better the solution). Furthermore, with nodal elements, vector quantities are 
represented by three functions, one for each component (in cartesian coordinates). The geometrical meaning is lost together with pull-back properties and the naturalness of the transformation method. The practical implementation is then much more cumbersome.

It is worth noting that the edge elements belong to the Hilbert space:

$$
L^{2}(\operatorname{curl}, \theta)=\left\{f \in L^{2}(\theta), \operatorname{curl} f \in L^{2}(\theta), n \times f=0 \text { on } \partial \theta\right\}
$$

and it has been proven [13] that for a given field $\varphi$ in $L^{2}(\operatorname{curl}, \theta)$, a sequence of Whitney edge elements $\varphi_{m}$ converges to $\varphi$ for the norm of $L^{2}(\operatorname{curl}, \theta)$, provided that the tetrahedra of the mesh tends to 0 in a given way. Concerning the node elements of $W^{0}$ it is classical that they tend towards a given function $\varphi$ in $H^{1}(\theta)$ : such a convergence therefore necessitates to place the study in an inadequate functional space $\left(H^{1}(\theta)\right)$, which leads to divergence penalization techniques.

\section{NUMERICAL IMPLEMENTATION}

Our discrete formulation leads to a generalized eigenvalue problem solved thanks to the Lanczos algorithm, which allows the computation of several eigenvalues and their associated eigenvectors for Hermitian matrices. In the Lanczos algorithm, spectral shifting is required to give access to a given part of the spectrum and/or to accelerate or even guarantee the convergence towards a given eigenvalue. The $(D R)$ relations give a useful practical estimate of the shift to be used to obtain the lowest frequency modes. The interested reader can get further details in [16-17].

\subsection{The Lanczos Algorithm}

The Lanczos algorithm is an iteration method on both eigenvectors and eigenvalues adapted to the resolution of generalized eigenvalue problems of the following form [16-17]:

$$
K x=k_{0}^{2} M x
$$

where the matrices $K$ and $M$ (generally called mass and stiffness matrices) are Hermitian and positive definite. The underlying concept of this method is that of the power algorithm which is the basis of all methods using iteration on eigenvectors. The first main property of the power algorithm holds in its convergence rate towards a given solution independent of the matrix size, which potentially makes it the ideal method for very large systems. The second one is that it makes it 
possible to limit the solving of the eigenvalue problem to the number of required solutions. One of the main drawbacks of the Lanczos method is its bad convergence rate for close eigenvalues. One way to tackle this problem is to use a spectral shift. Let us now introduce the principle of the Lanczos algorithm: it consists of generating a subspace including the system fundamental eigensolutions by inverse iteration on one starting vector called $x_{0}$. From the latter, we construct the Krylov sequence

$$
\left\{x_{0}, K^{-1} M x_{0},\left(K^{-1} M\right)^{2} x_{0}, \ldots\right\}
$$

the terms of which are made orthogonal to each other by a construction process of conjugate directions. Starting from an arbitrary vector $x_{0}$, the Lanczos algorithm consists of combining inverse iteration operations and orthogonalization of the successive iterates by applying the relation:

$$
c_{p+1} x_{p+1}=K^{-1} M x_{p}-a_{p} x_{p}-b_{p-1} x_{p-1},
$$

with coefficients $a_{p}, b_{p-1}$ and $c_{p+1}$ determined in such a way that:

$$
\left\{\begin{array}{l}
x_{p+1}^{t} M x_{j}=0, \quad j<p+1, \\
x_{p+1}^{t} M x_{p+1}=1 .
\end{array}\right.
$$

One can easily calculate coefficients $a_{p}$ and $b_{p-1}$ by imposing the orthogonality of $x_{p+1}$ with respect to $x_{p}$ and $x_{p-1}$ with the GramSchmidt algorithm. One get that:

$$
\left\{\begin{array}{l}
a_{p}=x_{p}^{t} M K^{-1} M x_{p}, \\
b_{p-1}=x_{p}^{t} M K^{-1} M x_{p-1},
\end{array}\right.
$$

the orthogonality of $x_{p+1}$ to $x_{p-2}, x_{p-3}$ is then deduced by recurrence thanks to:

$$
x_{p}^{t} M K^{-1} M x_{p-j}=x_{p}^{t} M\left(c_{p+1-j} x_{p+1-j}+c_{p-j} x_{p-j}+b_{p-j-1} x_{p-j-1}\right),
$$

which vanish as soon as $j \geq 2$.

Let us now analyse the difficulties of the Lanczos method. First, it must be noticed that the dominant modes of the system are very quickly extracted from the started vector so that, after a few iterations, numerical errors become of the same order of magnitude as the Lanczos base vectors. The result is that vectors $\left[x_{0}, \ldots, x_{p-2}\right]$, in relation to which orthogonality has been achieved, reappear in iterate $x_{p+1}$. We overcome this situation by reconstructing the direction's orthogonality 
by a Gram-Schmidt process, but it is obvious that we lose part of the method's benefit. Nevertheless, a selective re-orthogonalization process leads to a reduction of the cost of the operation.

Theoretically, multiple eigenvectors can not be extracted using the Lanczos method: indeed, the starting vector contains only a linear combination of those multiple modes, and this combination remains unchanged in the course of iterations. In practice, the result of the degeneration of the orthogonalization process is that multiple solutions reappear progressively. In the case of a dielectric waveguide of circular cross section, we know that the degeneracy of the modes induced by the symmetry is 2 . We numerically verify that the eigenvalues belonging to the discrete spectrum (those with physical meaning) are given twice (with ten significant figures), but their associated modes are different. Furthermore, we restart the algorithm with other starting vectors, therefore we can guarantee effective computation of all eigenvectors with a multiple eigenvalue.

Another difficulty is that of appearance of parasitic solutions or even skipping of solutions, which result from the degeneration of the orthogonalization process and a bad conditioning of matrices $K$ and $M$. To overcome this difficulty, we multiply the $M$ matrix by a factor $c^{2}$ ( $c$ being the celerity of light in vacuum): we then have two matrices $K$ and $c^{2} M$ with the same order coefficients, which induces a good starting vector. It is to be noticed that non-converged solutions can be rejected a posteriori by an error criterion [21].

To conclude this section, we want to say that the Lanczos method is extremely powerful for the extraction of eigenvalues and makes it possible to treat very large sparse systems. But its implementation is delicate and its use sometimes requires precautions. Nowadays, it is used in most finite element analysis codes.

\subsection{Validation of the Code}

Let us first note that the GetDP software [14] has been used to set up the finite element problem. We study the case of a low index step fiber of circular cross-section $\left(\varepsilon_{r}=1.25\right)$ to validate our method: it is then easy to compare our dispersion curve to that derived of weakcoupling assumptions [18]. In this case, the $H E_{1 m}$ modes have a small azimuthal variation and depend only on the radial position $\mathbf{r}$. There is therefore no preferred axis of symmetry in the circular crosssection. In this exceptional case, the transverse magnetic field can be directed so that it is everywhere parallel to one of an arbitrary pair of orthogonal directions. If we denote this pair of directions by $x$ - and $y$-axes, then there are two fundamental or $H E_{1 m}$ modes, one with its transverse magnetic field parallel to the $x$-direction, and the 


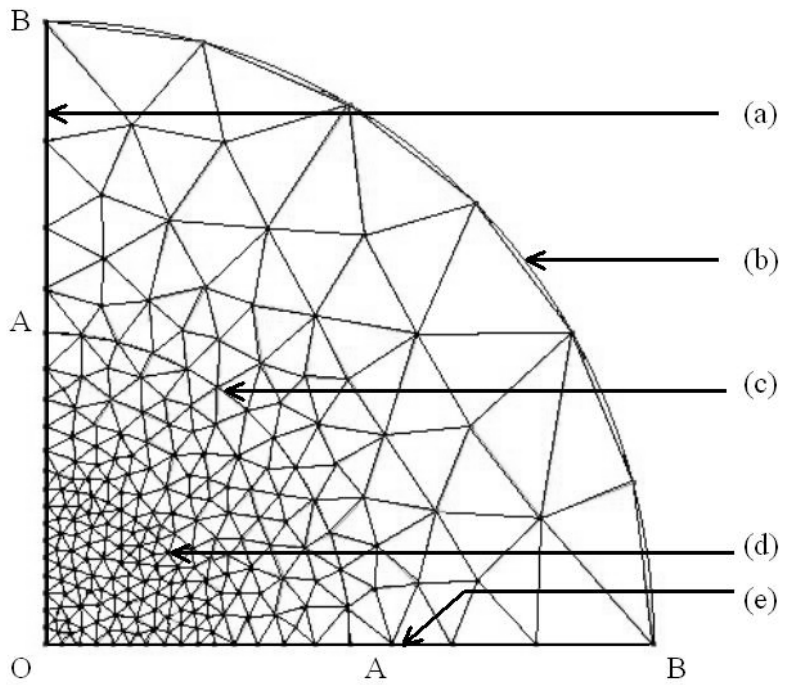

Figure 4. (a), (e) Projections of a plane of symmetry (Dirichlet condition) or antisymmetry (Neumann condition). (b) Exterior boundary of the corona rejected to infinity (Dirichlet boundary condition). (c) Interface between air and corona (tangential continuity of the Whitney elements). (d) Interface between core and air (tangential continuity of the Whitney elements).

other parallel to the $y$-direction. The symmetry also requires that the scalar propagation constants of each pair of modes are equal. This degenerescence of the modes highly depends upon the geometry of the waveguide cross-section. It is worth noting that if the cross-section is elliptical, the transverse magnetic field is everywhere parallel to the major and minor axes of the elliptical cross-section. As for the circular fiber there are two modes associated with this solution (one for each polarization). Because the cross-section is not circular, the propagation constants of these two modes will differ: the difference is due to polarization, or birefringence properties of the waveguide. In this sense, the propagation on noncircular waveguides is similar to propagation in anisotropic media. It is worth noting that with the exception of the fundamental $H E_{11}$ mode, every mode is cut off below a certain value of $V$ (see Section 2).

Thanks to the symmetry of the guide, we just mesh one fourth of the guide (we could have considered a smaller part of the guide): we take Dirichlet or Neumann boundary conditions on the $x$ and $y$ axis to get symmetric or antisymmetric modes versus these axes (Fig. 4). 


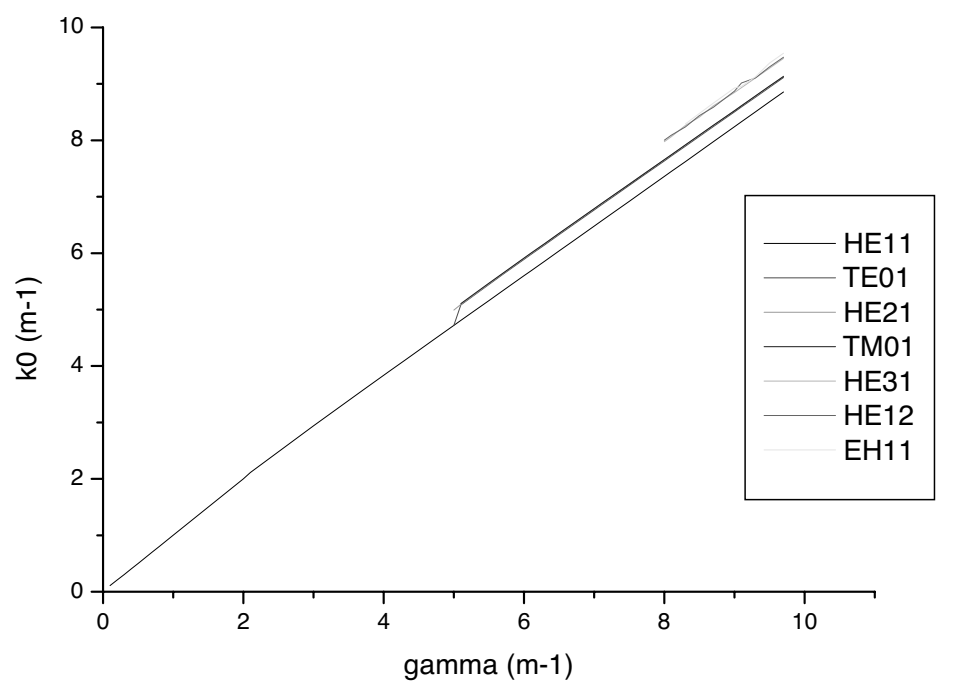

Figure 5. Dispersion curves of a fiber of circular cross-section with relative permittivity $\varepsilon_{r}=1.25$ and a unit radia.

This remark is of importance in the case of PCF structures: we only consider one fourth of the PCF since their crystal cladding have the square symmetry. Our validation is all the more significant that we obtain both the good dispersion curves (Fig. 5) and the associated expected transverse magnetic fields (Fig. 6) for all the modes [18]. Furthermore, if we choose the formulation with the electric field, our results strengthen those foreseen by B. Meys [15].

\subsection{Numerical Results for the PCF}

Let us first discuss the physics underlying photonic propagation in the first type of photonic crystal fibers (HPCF of glass with air holes), i.e., with a high index structural defect: we consider a waveguide of circular cross-section composed of a matrix of index 2 and 80 air holes periodically arranged. We achieve a numerical study of modes confined in specific parts of the high index region thanks to the crystal cladding: one can see on figure (Fig. 7) some propagating modes trapped in the high index core region of the fiber and one can see the analogous phenomenon on figure (Fig. 8) for modes confined outside the crystal cladding (and even inside-outside). The most popular physical interpretation is that the effective index for the crystal cladding is lower than that one of core and exterior regions and the modes therefore propagate in these regions. Such an effect is very 


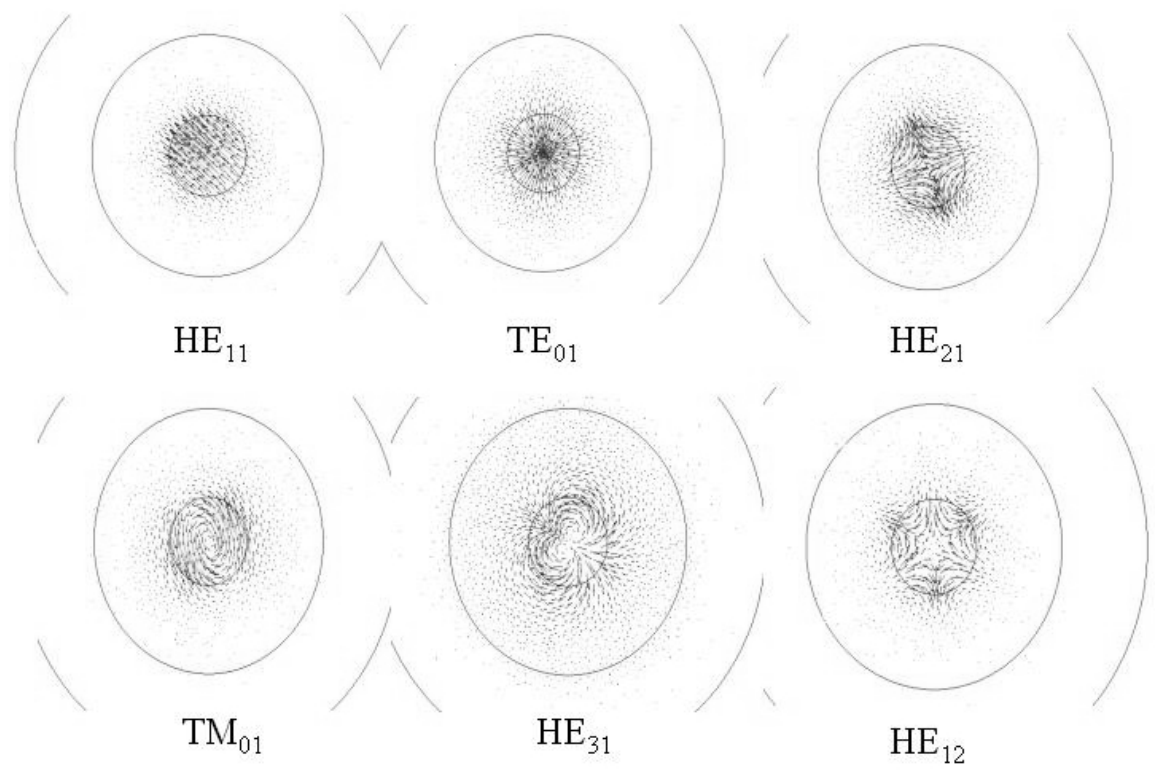

Figure 6. The four hybrid modes $H E_{11}, H E_{21}, H E_{31}, H E_{12}$ (ordered in increasing frequencies) and the two transverse modes $T E_{01}$ and $T M_{01}$ of the circular fiber of radius 1 with relative permittivity of $\varepsilon_{r}=1.25$ (corresponding to the dispersion curves of Fig. 5) for a propagation constant $\gamma=8 \mathrm{~m}^{-1}$. Their associated wave numbers are $k_{0}=7.38194,7.70039,7.99773,7.97468,7.62185$ and $7.73754 \mathrm{~m}^{-1}$.

similar to that of the classical waveguides. A second interpretation can be formulated: we consider the periodic assembly of air holes as independent fibers (although they are air holes) that are strongly coupled together. Let us assume that each isolated rod could support many distinct modes (obviously, these modes are purely complex since they cannot propagate in air holes), each with a different frequency $\omega$. When a large number of such rods (in our case 80) are placed in close proximity, they couple together, and each mode of the single rod opens up into a passband of modes of the composite structure (when the number of rods tends to infinity), each passband now covering a range of $\omega$ values. The passbands are separated by band gaps also known as photonic band gaps. The central high index defect would, if isolated, support a different set of waveguide modes, thanks to its different morphology. If the $\omega$ value of one of these modes falls within one of the bands of modes of the periodic cladding, this mode of the core will be coupled to the spatially extended modes of the periodic 

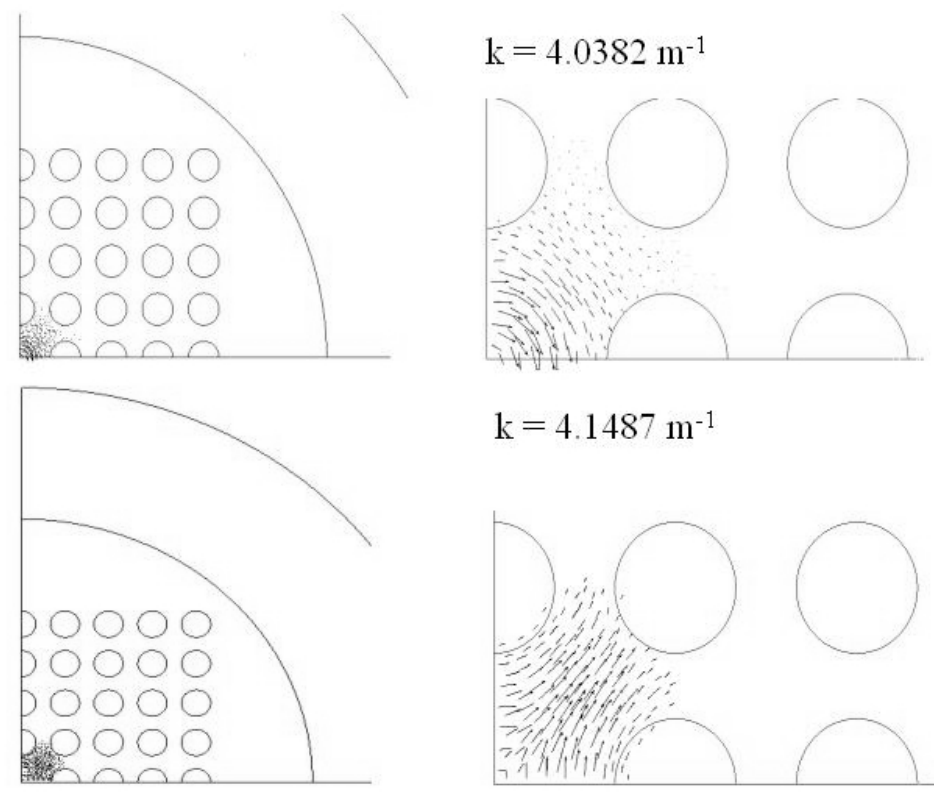

$$
\mathrm{k}=4.1487 \mathrm{~m}^{-1}
$$

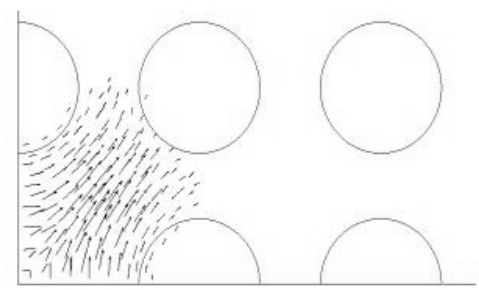

Figure 7. Magnetic fields and their associated wave numbers $k$ in a circular HPCF (PCF type 1) of index 2 with 80 unit radius air holes for a propagation constant $\gamma=8 \mathrm{~m}^{-1}$.

cladding (everywhere in high index). However, if one of the modes of the core region falls in between the passbands of the fully periodic lattice region, then this mode is localized within the core and forms a PBG guided mode. Thus, at some wavelengths, there is a mode trapped within the core (resp. outside the crystal cladding), whereas at other wavelengths modes are extending between the fibers (they cannot propagate in air holes).

Let us now introduce some qualitative insight into the nature of the guided modes of a low-index PCF structure: (LPCF with silica rods). Although rigorous, our numerical study holds only for the nondissipative modes. The localized mode is a kind of "leaky mode," that is a dissipative mode, and is therefore associated to complex frequencies. To look for such a mode necessitates to investigate the case of complex propagation constant $\gamma$. For this, it seems more natural to work with a fixed frequency $\omega$ and look for $\gamma$ : we are thus led to a nonlinear generalized eigenvalue problem with non-hermitian matrices. Such a "spectral problem" could be numerically solved thanks to an algorithm of non linear inverse iterations. This algorithm can indeed 


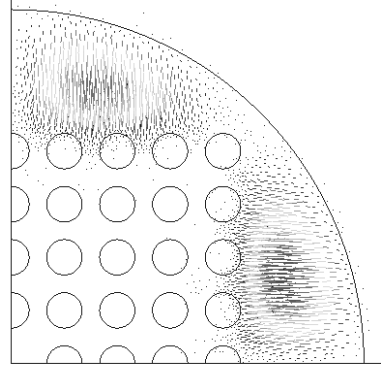

$\mathrm{k}=3.3574 \mathrm{~m}^{-1}$

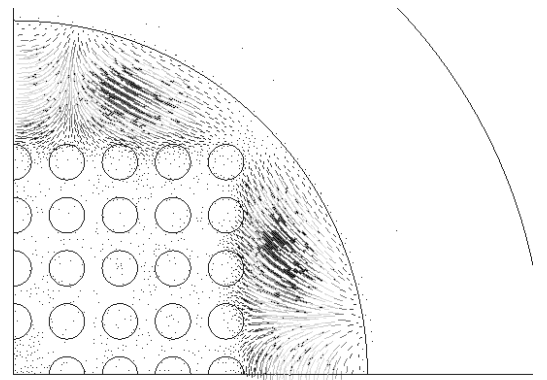

$\mathrm{k}=3.3689 \mathrm{~m}^{-1}$

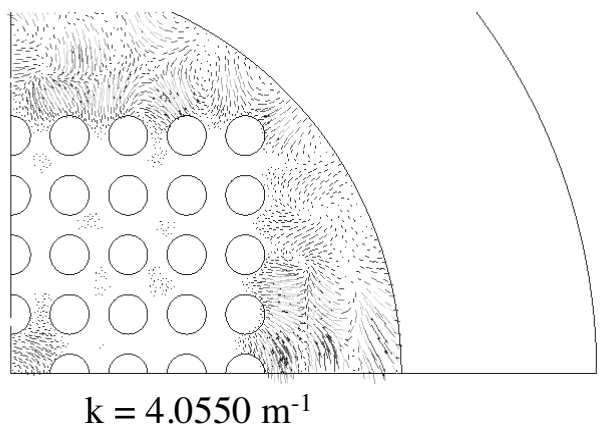

Figure 8. Magnetic fields and their associated wave numbers $k$ in a circular HPCF (PCF type 1) of index 2 with 80 unit radius air holes for a propagation constant $\gamma=8 \mathrm{~m}^{-1}$.

solve our generalized eigenvalue problems which takes the form:

$$
K(\gamma) X=\omega^{2} M X,
$$

where the matrix $K$ is complex non hermitian and depends non-linearly on $\gamma$ and $M$ is a complex hermitian matrix. The main difficulty is that this algorithm only gives one eigenvalue and its associated eigenvector per calculus. Therefore, we do not investigate the leaky modes in this paper. Nevertheless, we achieve a numerical study of modes propagating in fibers of index 2 in a crystal cladding. We clearly see that such modes propagate in each fiber independently of the other fibers (which is far from being obvious) (Fig. 9): these modes have the same frequencies and symmetries (up to a phasis) than the ones of a single fiber of unit radius and relative permittivity $\varepsilon=4$. Obviously, these modes are identical in each fiber up to a phasis. Such a result can although be interpreted as modes belonging to the Bloch spectrum: in the Bloch wave decomposition, one only considers a fiber in a unit cell $Y$ with quasi-periodicity conditions (up to a phasis). 


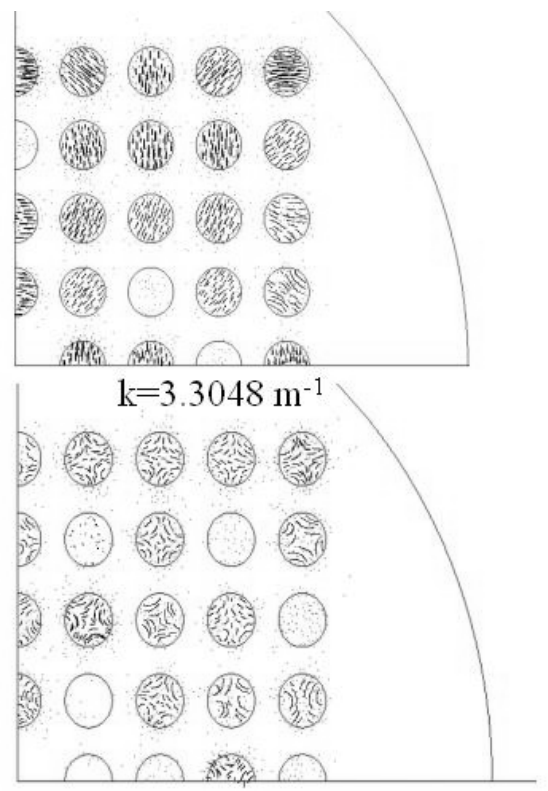

$\mathrm{k}=3.3574 \mathrm{~m}^{-1}$

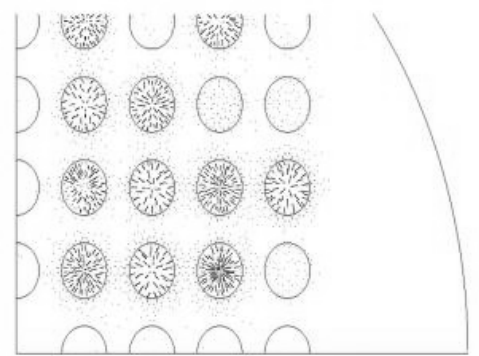

$\mathrm{k}=3.3086 \mathrm{~m}^{-1}$

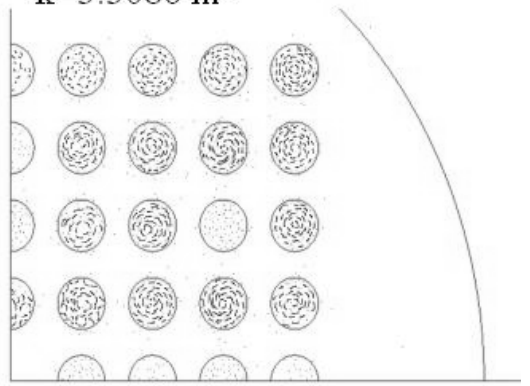

$\mathrm{k}=3.3689 \mathrm{~m}^{-1}$

Figure 9. Magnetic fields and their associated wave numbers $k$ in a circular LPCF (PCF type 2) of index 1 with 80 unit radius rods of index 3 for a propagation constant $\gamma=5 \mathrm{~m}^{-1}$.

\section{CONCLUSION}

As a conclusion, we want to say a few words about the physical aspect of the study. Due to the vector treatment of the problem, we achieve a rigorous numerical study of the physical phenomena arising in propagation of modes in a photonic crystal fiber. We first compare the numerical results of the finite element scheme with classical ones: for this, we study the dispersion curves of a dielectric circular waveguide. We then study two types of photonic crystal fibers of finite cross section. In the first type of PCF (HPCF), we numerically characterize modes which propagates thanks to the photonic band gap effect in a region of high index surrounding a crystal cladding (fiber of silica with a periodic assembly of air holes). In the second type of PCF (LPCF), we numerically show some propagating modes confined in each rod (silica) of the crystal cladding and which have all the properties of the modes of a circular fiber: the frequencies and associated modes of such fibers do not depend on the existence of the other fibers and 
are thus called local modes. We are working at the present time on the generalization of the method to the computation of leaky modes. Other remarkable properties of PCF are that linearly polarized light coupled into the fiber parallel to one axis emerges linearly polarized and parallel to the same axis even if the fiber is bent or twisted. An analogous phenomena is even observed concerning the loss of energy induced by the bends: unlike classical optical waveguides, the PCF can propagate light without loss of energy in the bends, thanks to the PBG effect. Such a modelling could be achieved with our formalism, thanks to adequate geometrical transformation (by the use of pull-back properties of the edge-elements).

\section{ACKNOWLEDGMENT}

This work is supported by the Société Européenne de Systèmes Optiques and by the Région Provence Alpes Côte d'Azur.

\section{REFERENCES}

1. Birks, T. A., J. C. Knight, and P. St. J. Russell, "Endlessly singlemode photonic crystal fiber," Optics Letters, Vol. 22, No. 13, 961963, July 1997.

2. Knight, J. C., T. A. Birks, R. F. Cregan, P. St. J. Russell, and J. P. de Sandro, "Large mode area photonic crystal fibre," Electronics Letters, Vol. 34, No. 13, 1347-1348, June 1998.

3. Knight, J. C., T. A. Birks, P. St. J. Russell, and J. P. de Sandro, "Properties of photonic crystal fiber and the effective index model," JOSA A, Vol. 15, 748-, 1998.

4. Broeng, J., D. Mogilevstev, S. E. Barkou, and A. Bjarklev, "Photonic crystal fibers: a new class of optical waveguides," Optical Fiber Technology, 1999.

5. Knight, J. C., J. Broeng, T. A. Birks, and P. St. J. Russell, "Photonic band gap guidance in optical fibers," Science, Vol. 282, 1476-1478, November 1998.

6. Whitney, H., Geometric Integration Theory, Princeton Univ. Press, Princeton, 1957.

7. Guenneau, S., A. Nicolet, F. Zolla, C. Geuzaine, and B. Meys, "A finite element formulation for spectral problems in optical fibers," Compel, Vol. 20, No. 1, 120-131, 2001.

8. Bonnet, A.-S., "Analyse mathématique de la propagation des modes guidés dans les fibres optiques," Ph.D. Thesis, Paris VI, 1988 . 
9. Reed, M. and B. Simon, Methods of Modern Mathematical Physics, Vol. 1, Academic Press, New York, 1978.

10. Bossavit, A., "Solving Maxwell equations in a closed cavity, and the question of spurious modes," IEEE Transactions on Magnetics, Vol. 26, No. 2, 1990.

11. Bossavit, A., "Electromagnétisme en vue de la modélisation," Mathématiques et Applications, Vol. 14, Springer Verlag, 1993.

12. Nicolet, A., J-F. Remacle, B. Meys, A. Genon, and W. Legros, "Transformation methods in computational electromagnetism," $J$. Appl. Phys., Vol. 75, No. 10, 1994.

13. Dodziuk, J., "Finite-difference approach to the Hodge theory of harmonic forms," Amer. J. Math., Vol. 98, 79-104, 1976.

14. Dular, P., C. Geuzaine, F. Henrotte, and W. Legros, "A general environment for the treatment of discrete problems and its application to the finite element method," IEEE Transactions on Magnetics, Vol. 34, No. 5, 3395-3398, 1998.

15. Meys, B., "Modélisation des champs électromagnétiques aux hyperfréquences par la méthode des éléments finis, application au problème du chauffage diélectrique," Ph.D. Thesis, Université de Liège, 1999.

16. Saad, Y., Numerical Methods for Large Eigenvalue Problems, Manchester Univ. Press, Ser. in alg. arch. for adv. sci. comp., 1991.

17. Geradin, M. and D. Rixen, Mechanical Vibrations: Theory and Applications to Structural Dynamics, John Wiley and Son, 2nd edition, 1997.

18. Snyder, A. W. and J. D. Love, Optical Waveguide Theory, Chapman and Hall, New York, 1983.

19. Petit, R., Ondes Electromagnétiques en Radioélectricité et en Optique, Masson, 1993.

20. Vassallo, C., Théorie des Guides d'Ondes en Electromagnétisme, CNET, Eyrolles editions, 1985.

21. Guenneau, S., "Homogénéisation des quasi-cristaux et analyse des modes dans des fibres optiques de type cristal photonique," Ph.D. Thesis, Université de Provence, 2001. 